Homology, Homotopy and Applications, vol.12(1), 2010, pp.157-185

\title{
RATIONAL GENERALIZED INTERSECTION HOMOLOGY THEORIES
}

\author{
MARKUS BANAGL \\ (communicated by Michael A. Mandell)
}

\begin{abstract}
Given a spectrum $E$, we investigate the theory that associates to a stratified pseudomanifold the tensor product of its Goresky-MacPherson intersection homology with the rationalized coefficients of $E$. The viewpoint adopted in this paper is to express this theory as the homotopy groups of a spectrum associated to the pseudomanifold and $E$. The relation is given by an Atiyah-Hirzebruch formula. Properties such as topological invariance, generalized Poincaré duality, behavior under small resolution, products, cohomology operations, and the Künneth spectral sequence are then discussed from that viewpoint. Moreover, we consider self-dual generalized (co)homology theories on spaces that need not satisfy the Witt condition. Local calculations and a sample calculation of the rational intersection $k u$-theory of a certain singular Calabi-Yau 3-fold are carried out. We employ the framework of $S$-algebras and modules over Eilenberg-MacLane spectra due to Elmendorf, Kriz, Mandell and May.
\end{abstract}

\section{Introduction}

Let $E$ be any $S$-module in the sense of [EKMM97]. (Any CW spectrum, for example, is homotopy equivalent to an $S$-module.) Let $\bar{p}$ be any perversity in the sense of [GM83] and let $I H_{*}^{\bar{p}}(-; \mathbb{Q})$ denote intersection homology as defined in [GM83]. To any $n$-dimensional, topological pseudomanifold $X$, one can assign the homology groups

$$
\bigoplus_{i+j=k} I H_{i}^{\bar{p}}(X ; \mathbb{Q}) \otimes_{\mathbb{Q}}\left(\pi_{j}(E) \otimes_{\mathbb{Z}} \mathbb{Q}\right)
$$

and the cohomology groups

$$
\prod_{i-j=k} \operatorname{Hom}_{\mathbb{Q}}\left(I H_{i}^{\bar{p}}(X ; \mathbb{Q}), \pi_{j}(E) \otimes_{\mathbb{Z}} \mathbb{Q}\right) .
$$

The author was in part supported by a research grant of the Deutsche Forschungsgemeinschaft. Received September 22, 2008, revised August 12, 2009; published on March 19, 2010.

2000 Mathematics Subject Classification: 55N33, 55N20, 55P42.

Key words and phrases: intersection homology, generalized homology theories, spectra, stable homotopy theory, self-dual sheaves, stratified spaces, singularities.

This article is available at http://intlpress.com/HHA/v12/n1/a10

Copyright (c) 2010, International Press. Permission to copy for private use granted. 
The goal of this paper is to express these groups as the homotopy groups of a spectrum and to investigate their properties from this point of view. Let $H \mathbb{Q}$ denote an Eilenberg-MacLane spectrum $H \mathbb{Q}=K(\mathbb{Q}, 0)$, constructed as a commutative $S$ algebra. Then $E \mathbb{Q}=E \wedge_{S} H \mathbb{Q}$ is an $H \mathbb{Q}$-module and we set

$$
I E_{k}^{\bar{p}}(X ; \mathbb{Q})=\pi_{k}\left(E \mathbb{Q} \wedge_{H \mathbb{Q}} \Phi I C_{\bullet}^{\bar{p}}(X ; \mathbb{Q})\right)
$$

and

$$
I E_{\bar{p}}^{k}(X ; \mathbb{Q})=\pi_{-k}\left(F_{H \mathbb{Q}}\left(\Phi I C_{\bullet}^{\bar{p}}(X ; \mathbb{Q}), E \mathbb{Q}\right)\right)
$$

(Definition 2.5). Here $\Phi$ is a functor, described in Section 2, that is built using Brown's representability theorem and yields an $H \mathbb{Q}$-module spectrum, and $F_{H \mathbb{Q}}$ denotes the function $H \mathbb{Q}$-module. Throughout the paper, we refer to the former groups as the rational perversity $\bar{p}$ intersection E-homology of $X$ and to the latter groups as the rational perversity $\bar{p}$ intersection E-cohomology of $X$. Proposition 8.1 shows that $I E_{k}^{\bar{p}}(X ; \mathbb{Q})$ agrees with $(1)$ and Proposition 8.2 shows that $I E_{\bar{p}}^{k}(X ; \mathbb{Q})$ agrees with $(2)$. Not using this identification, but proceeding directly from the definition of $I E_{k}^{\bar{p}}(X ; \mathbb{Q})$ and $I E_{\bar{p}}^{k}(X ; \mathbb{Q})$ as homotopy groups of spectra, we show that the properties listed below are satisfied. Most of these properties on the level of homology groups can be readily established from (1) and (2) as well, but our proofs are fashioned with the hope in mind that they might facilitate future purely spectrum-level arguments, e.g., when investigating other rings.

1. If $E=H \mathbb{Z}$ is an Eilenberg-MacLane spectrum $K(\mathbb{Z}, 0)$, then

$$
I E_{k}^{\bar{p}}(X ; \mathbb{Q}) \cong I H_{k}^{\bar{p}}(X ; \mathbb{Q}) .
$$

2. If $X$ is compact and nonsingular, then

$$
I E_{k}^{\bar{p}}(X ; \mathbb{Q}) \cong E_{k}(X) \otimes \mathbb{Q} .
$$

3. If $f: Y \rightarrow X$ is a small resolution of singularities between compact, complex algebraic varieties, then

$$
I E_{k}^{\bar{m}}(X ; \mathbb{Q}) \cong E_{k}(Y) \otimes \mathbb{Q}
$$

where $\bar{m}$ denotes the lower middle perversity. As a corollary we obtain the result that any two small resolutions of $X$ have isomorphic rational $E$-homology.

4. Topological Invariance: Any homeomorphism $f: X \rightarrow Y$ between topological pseudomanifolds induces isomorphisms

$$
I E_{*}^{\bar{p}}(X ; \mathbb{Q}) \cong I E_{*}^{\bar{p}}(Y ; \mathbb{Q}), \quad I E_{\bar{p}}^{*}(X ; \mathbb{Q}) \cong I E_{\bar{p}}^{*}(Y ; \mathbb{Q})
$$

for any $S$-module $E$.

5. Poincaré Duality: For compact, oriented $X^{n}$, there is a generalized Poincaré duality isomorphism

$$
I E_{n-k}^{\bar{q}}(X ; \mathbb{Q}) \cong I E_{\bar{p}}^{k}(X ; \mathbb{Q})
$$

for any $S$-module $E$ and complementary perversities $\bar{p}$ and $\bar{q}$. 
6. External Products: Let $X$ and $Y$ be compact. Let $\bar{p}$ be a perversity satisfying the "Künneth condition"

$$
\bar{p}(k)+\bar{p}(l) \leqslant \bar{p}(k+l) \leqslant \bar{p}(k)+\bar{p}(l)+2
$$

for all $k, l$ (for example, $\bar{p}=\bar{m}$ ). Let $E$ be an $S$-algebra. Then there is an external intersection $E$-cohomology product

$$
I E_{\bar{p}}^{*}(X ; \mathbb{Q}) \otimes_{\mathbb{Q}} I E_{\bar{p}}^{*}(Y ; \mathbb{Q}) \longrightarrow I E_{\bar{p}}^{*}(X \times Y ; \mathbb{Q}) .
$$

7. Internal Products: Let $X$ be compact, let $\bar{p}$ satisfy the above Künneth condition, and let $\bar{q}$ be a perversity such that

$$
\bar{q}(k)+k \leqslant \bar{p}(2 k)
$$

for all $k \geqslant 2$. (There are plenty of such pairs $(\bar{p}, \bar{q})$.) Then there is an internal (cup) product

$$
I E_{\bar{p}}^{*}(X ; \mathbb{Q}) \otimes_{\mathbb{Q}} I E_{\bar{p}}^{*}(X ; \mathbb{Q}) \stackrel{\cup}{\longrightarrow} I E_{\bar{q}}^{*}(X ; \mathbb{Q}) .
$$

8. IE-cohomology operations: For an arbitrary pseudomanifold $X$ and arbitrary perversity $\bar{p}$, every element $\alpha$ of the algebra of operations $(E \mathbb{Q})_{H \mathbb{Q}}^{*}(E \mathbb{Q})$ defines a cohomology operation

$$
\alpha: I E_{\bar{p}}^{*}(X ; \mathbb{Q}) \longrightarrow I E_{\bar{p}}^{*}(X ; \mathbb{Q}) .
$$

9. Künneth spectral sequence: Let $\bar{p}$ be a perversity satisfying the above Künneth condition. For any $S$-algebra $E$ and compact pseudomanifolds $X, Y$, there is a spectral sequence

$$
E_{p, q}^{2}=\operatorname{Tor}_{p, q}^{E_{*} \otimes \mathbb{Q}}\left(I E_{*}^{\bar{p}}(X ; \mathbb{Q}), I E_{*}^{\bar{p}}(Y ; \mathbb{Q})\right) \Longrightarrow I E_{p+q}^{\bar{p}}(X \times Y ; \mathbb{Q}) .
$$

The properties obtained in this paper may serve as a yardstick for potential future constructions of generalized intersection homology over more general rings. When such a putative theory is tensored with the rationals, it should satisfy all the above properties. Note that integral theories $I E_{*}^{\bar{p}}(X ; \mathbb{Z}), I E_{\bar{p}}^{*}(X ; \mathbb{Z})$ satisfying the above properties cannot exist on the full class of oriented pseudomanifolds considered here: Let $M^{m}$ be an oriented smooth closed connected manifold and let $V \rightarrow M$ be an oriented $m$-plane vector bundle over $M$ with nonzero Euler number $e$. The Thom space $X^{n}=V \cup\{\infty\}, n=2 m$, is a pseudomanifold with an isolated singularity at $\infty$. Its middle dimensional integral intersection homology is $I H_{m}^{\bar{m}}(X ; \mathbb{Z})=\mathbb{Z}$ and the determinant of the intersection form is $e$. So if $e \neq \pm 1$, then the intersection form is not unimodular; see [GM80]. While the passage from $E$ to $E \mathbb{Q}$ naturally forgets a fair amount of information about $E$, enough is preserved that the theories $I E_{*}^{\bar{p}}(X ; \mathbb{Q})$, $I E_{\bar{p}}^{*}(X ; \mathbb{Q})$ are expected to have interesting applications, e.g., in equivariant situations; see [CSW91].

In Section 10, we give the local calculations for intersection $E$-homology; that is, we compute $I E_{*}^{\bar{p}}(U ; \mathbb{Q})$ for a distinguished neighborhood $U$ in a stratified pseudomanifold.

In Section 11, we construct self-dual E-homology theories on oriented pseudomanifolds $X$ that do not necessarily satisfy the Witt condition. On such spaces, middle perversity intersection homology is not self-dual. In order to obtain self-dual theories, 
which are important (for example) in defining characteristic classes, one may employ Lagrangian structures in the sense of [Ban02], [Ban06]; see also [Ban07] for an exposition. Let $E$ be any $S$-module and $\mathcal{L}$ a Lagrangian structure along the strata of odd codimension of $X$. We construct rational $\mathcal{L}$-intersection $E$-homology groups $I E_{k}^{\mathcal{L}}(X ; \mathbb{Q})$ and rational $\mathcal{L}$-intersection E-cohomology groups $I E_{\mathcal{L}}^{k}(X ; \mathbb{Q})$ such that there exists a factorization

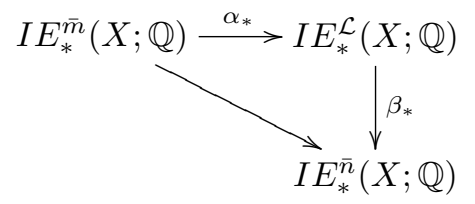

of the canonical morphism and a commutative duality square

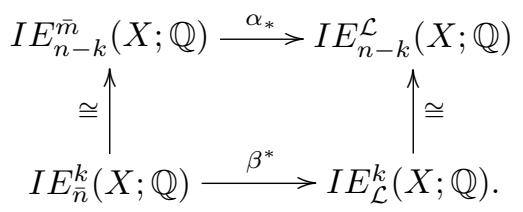

We conclude with a sample calculation: In Section 12, we compute the intersection $k u$-theory of a certain singular Calabi-Yau 3-fold.

The backbone of our method is an equivalence of categories between the stable homotopy category of module spectra over an Eilenberg-MacLane spectrum and the derived category of chain complexes; see [Rob87], [Rob83], [EKMM97]. The reason why our method does not work over the integers is that we make systematic use of Serre's theorem which states that rational homology is the same as rational stable homotopy.

Technically, we work in the framework of $S$-algebras and modules over them as developed in [EKMM97]. We follow the notation of [EKMM97] closely throughout the paper. As far as intersection homology is concerned, our notation and indexing conventions follow [GM83].

\section{The Basic Construction}

Let $\mathcal{D}(\mathbb{Q})$ denote the derived category obtained from the homotopy category of chain complexes of rational vector spaces by localizing at the quasi-isomorphisms. Let $H \mathbb{Q}$ denote an Eilenberg-MacLane spectrum $H \mathbb{Q}=K(\mathbb{Q}, 0)$, constructed as a commutative $S$-algebra. Let $\mathcal{D}(H \mathbb{Q})$ denote the homotopy category of $H \mathbb{Q}$-modules localized at the weak equivalences. Let us briefly recall the equivalence of categories

$$
C h: \mathcal{D}(H \mathbb{Q}) \rightleftarrows \mathcal{D}(\mathbb{Q}): \Phi .
$$

By approximation by cell modules and by Whitehead's theorem, the category $\mathcal{D}(H \mathbb{Q})$ is equivalent to the homotopy category of $\mathrm{CW} H \mathbb{Q}$-modules and cellular maps. Let $M$ be a $C W H \mathbb{Q}$-module. Define a chain complex $C h(M)$ of abelian groups by setting $C h(M)_{n}=\pi_{n}\left(M^{n} / M^{n-1}\right)$ and letting the differential $\partial_{n}: C h(M)_{n} \rightarrow C h(M)_{n-1}$ be the connecting homomorphism of the triple $\left(M^{n}, M^{n-1}, M^{n-2}\right)$. A cellular map of 
$H \mathbb{Q}$-modules induces a map of chain complexes and a cellular homotopy induces a chain homotopy. Thus, we obtain a functor

$$
C h: \mathcal{D}(H \mathbb{Q}) \longrightarrow \mathcal{D}(\mathbb{Q}) .
$$

To construct the inverse equivalence $\Phi$, consider, for a fixed chain complex $C_{\bullet}$, the functor $k$ on $\mathcal{D}(H \mathbb{Q})$ given by $k(M)=\mathcal{D}(\mathbb{Q})(C h(M), C \bullet)$. Since $k$ satisfies the wedge and Mayer-Vietoris axioms, Brown's representability theorem asserts that $k$ is represented by an $H \mathbb{Q}$-module spectrum $\Phi\left(C_{\bullet}\right)$. Thus, we obtain a covariant functor

$$
\Phi: \mathcal{D}(\mathbb{Q}) \longrightarrow \mathcal{D}(H \mathbb{Q})
$$

and an adjunction

$$
\mathcal{D}(\mathbb{Q})\left(C h(M), C_{\bullet}\right) \cong \mathcal{D}(H \mathbb{Q})\left(M, \Phi\left(C_{\bullet}\right)\right) .
$$

The functor $\Phi$ converts tensor products into smash products,

$$
\Phi\left(C_{\bullet} \otimes_{\mathbb{Q}} D_{\bullet}\right) \cong \Phi C_{\bullet} \wedge_{H \mathbb{Q}} \Phi D_{\bullet},
$$

and converts homology groups into homotopy groups,

$$
H_{*}\left(C_{\bullet}\right) \cong \pi_{*}\left(\Phi C_{\bullet}\right) .
$$

Formula (4) implies

$$
\pi_{*}(M) \cong H_{*}(C h(M))
$$

for any $H \mathbb{Q}$-module $M$, because

$$
\pi_{*}(M) \cong \pi_{*}(\Phi C h(M)) \cong H_{*}(C h(M)) .
$$

In $\mathcal{D}(\mathbb{Q}$ ), any chain complex is isomorphic to its homology complex (with zero differentials). Thus, $C h(M) \cong H_{\bullet}(C h(M)) \cong \pi_{\bullet}(M)$. This shows that one may think of $\pi_{\bullet}$ as inducing the equivalence $\mathcal{D}(H \mathbb{Q}) \simeq \mathcal{D}(\mathbb{Q})$.

More generally, the functor $C h$ can be defined on CW $R$-modules $M$ for any connective $S$-algebra $R$. In this situation, $C h(M)_{n}=\pi_{n}\left(M^{n} / M^{n-1}\right)$ is a $\pi_{0}(R)$-module. If $R$ is commutative and $M, N$ are CW $R$-modules, then $M \wedge_{R} N$ is a CW $R$-module and

$$
C h\left(M \wedge_{R} N\right) \cong C h(M) \otimes_{\pi_{0}(R)} C h(N) .
$$

When $X$ is a based cell complex with skeletal filtration $\left\{X^{n}\right\}_{n \geqslant 0}$ and base-point $b$, then we always set $X^{n}=\{b\}$ for $n<0$. With this convention, $H_{0}\left(X^{0}, X^{-1}\right)=$ $\widetilde{H}_{0}\left(X^{0}\right)$ and $H_{n}\left(X^{n}, X^{n-1}\right)=0$ for $n<0$. Let $\widetilde{C}_{\bullet}(X)$ denote the integral cellular chain complex of $X$ given by $\widetilde{C}_{n}(X)=H_{n}\left(X^{n}, X^{n-1}\right), n \in \mathbb{Z}$. Our filtration convention implies $H_{*}\left(\widetilde{C}_{\bullet}(X)\right)=\widetilde{H}_{*}(X)$, the reduced homology of $X$. Let $\mathbb{Q}[0] \in \mathcal{D}(\mathbb{Q})$ denote the chain complex concentrated in degree 0 with $\mathbb{Q}$ in that degree, so that we may assume $H \mathbb{Q}=\Phi(\mathbb{Q}[0])$. We write $\widetilde{C} \bullet(X ; \mathbb{Q})=\widetilde{C}_{\bullet}(X) \otimes_{\mathbb{Z}} \mathbb{Q}$ for the $\mathbb{Q}$-coefficient cellular chain complex of $X$. To motivate our definition of generalized intersection homology, we make the following observation:

Proposition 2.1. Let $X$ be a based $C W$ complex and let $E$ be an $S$-module. Then

$$
E_{*}\left(\Sigma^{\infty} X\right) \otimes \mathbb{Q} \cong E_{*}(\Phi \widetilde{C} \cdot(X ; \mathbb{Q})) .
$$


Proof. We regard the suspension spectrum $\Sigma^{\infty} X$ as an $S$-module. In $\mathcal{D}(\mathbb{Q})$, any chain complex is isomorphic to its homology complex (with zero differentials). Thus,

$$
C h\left(\Sigma^{\infty} X \wedge_{S} H \mathbb{Q}\right) \cong H_{\bullet}\left(C h\left(\Sigma^{\infty} X \wedge_{S} H \mathbb{Q}\right)\right) \text { and } \widetilde{C}_{\bullet}(X ; \mathbb{Q}) \cong H_{\bullet}\left(\widetilde{C}_{\bullet}(X ; \mathbb{Q})\right)
$$

in $\mathcal{D}(\mathbb{Q})$. Composing these isomorphisms with

$$
\begin{aligned}
H_{\bullet}\left(C h\left(\Sigma^{\infty} X \wedge_{S} H \mathbb{Q}\right)\right) & \cong \pi_{\bullet}\left(\Sigma^{\infty} X \wedge_{S} H \mathbb{Q}\right) \\
& \cong(H \mathbb{Q}) \bullet\left(\Sigma^{\infty} X\right) \cong \widetilde{H}_{\bullet}(X ; \mathbb{Q})=H_{\bullet}\left(\widetilde{C}_{\bullet}(X ; \mathbb{Q})\right),
\end{aligned}
$$

we obtain an isomorphism

$$
C h\left(\Sigma^{\infty} X \wedge_{S} H \mathbb{Q}\right) \stackrel{\simeq}{\longrightarrow} \widetilde{C}_{\bullet}(X ; \mathbb{Q}) .
$$

This isomorphism induces an isomorphism

$$
\alpha: \Sigma^{\infty} X \wedge_{S} H \mathbb{Q} \cong \Phi C h\left(\Sigma^{\infty} X \wedge_{S} H \mathbb{Q}\right) \stackrel{\simeq}{\longrightarrow} \Phi\left(\widetilde{C}_{\bullet}(X ; \mathbb{Q})\right)
$$

in $\mathcal{D}(H \mathbb{Q})$. It follows that

$$
1_{E} \wedge_{S} \alpha: E \wedge_{S} \Sigma^{\infty} X \wedge_{S} H \mathbb{Q} \stackrel{\simeq}{\longrightarrow} E \wedge_{S} \Phi(\widetilde{C} \bullet(X ; \mathbb{Q}))
$$

is an isomorphism in $\mathcal{D}(H \mathbb{Q})$ as well, because the derived smash product $E \wedge_{S} M$, where $M$ is any $S$-module, is by definition $E \wedge_{S} \Gamma M$, with $\Gamma$ the cell approximation functor, and for $M=\Sigma^{\infty} X \wedge_{S} H \mathbb{Q}, M^{\prime}=\Phi\left(\widetilde{C}_{\bullet}(X) \otimes_{\mathbb{Z}} \mathbb{Q}[0]\right)$ there is a commutative square

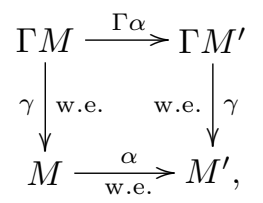

showing that $\Gamma \alpha$ is a weak equivalence; hence, by Whitehead's theorem, a homotopy equivalence. Then $1_{E} \wedge_{S} \alpha$ is represented by the homotopy equivalence $1_{E} \wedge_{S} \Gamma \alpha$. On homotopy groups, this homotopy equivalence induces

$$
\pi_{*}\left(E \wedge_{S} \Sigma^{\infty} X \wedge_{S} H \mathbb{Q}\right) \cong \pi_{*}\left(E \wedge_{S} \Phi(\widetilde{C} \bullet(X ; \mathbb{Q}))\right) .
$$

A representative $h: S \rightarrow H \mathbb{Z}$ of a generator of $\pi_{0}(H \mathbb{Z})=\mathbb{Z}$ induces, by Serre's theorem, an equivalence $S \mathbb{Q} \stackrel{\simeq}{\longrightarrow} H \mathbb{Q}$, where $S \mathbb{Q}$ denotes the Moore spectrum for $\mathbb{Q}$, constructed as an $S$-module. Using this equivalence, we get

$$
\begin{aligned}
E_{*}\left(\Sigma^{\infty} X\right) \otimes \mathbb{Q} & =E_{*}\left(\Sigma^{\infty} X \wedge_{S} S \mathbb{Q}\right) \\
& =\pi_{*}\left(E \wedge_{S} \Sigma^{\infty} X \wedge_{S} S \mathbb{Q}\right) \\
& \cong \pi_{*}\left(E \wedge_{S} \Sigma^{\infty} X \wedge_{S} H \mathbb{Q}\right) \\
& \cong \pi_{*}\left(E \wedge_{S} \Phi(\widetilde{C} \bullet(X ; \mathbb{Q}))\right) \\
& =E_{*}(\Phi \widetilde{C} \bullet(X ; \mathbb{Q})) .
\end{aligned}
$$

As usual, we shall henceforth write $\widetilde{E}_{*}(X):=E_{*}\left(\Sigma^{\infty} X\right)$ for a space $X$. For unreduced $E$-homology we obtain: 
Corollary 2.2. Let $X$ be a based $C W$ complex and let $E$ be an $S$-module. Then

$$
E_{*}(X) \otimes \mathbb{Q} \cong E_{*}(\Phi C \bullet(X ; \mathbb{Q})),
$$

where $C \bullet(X ; \mathbb{Q})$ is the (unreduced) rational cellular chain complex of $X$.

Proof. Let $X_{+}=X / \varnothing$ denote the union of $X$ with a disjoint base-point. Then

$$
C_{\bullet}(X)=\widetilde{C}_{\bullet}\left(X_{+}\right)
$$

and, using Proposition 2.1,

$$
\begin{aligned}
E_{*}(X) \otimes \mathbb{Q} & =\widetilde{E}_{*}\left(X_{+}\right) \otimes \mathbb{Q} \\
& \cong E_{*}\left(\Phi \widetilde{C} \cdot\left(X_{+} ; \mathbb{Q}\right)\right) \\
& =E_{*}(\Phi C \bullet(X ; \mathbb{Q})) .
\end{aligned}
$$

In the sheaf theoretic approach to intersection homology, it is customary to work with cochain complexes rather than chain complexes. The functor ${ }^{-}: \mathcal{C H}^{\bullet} \rightarrow \mathcal{C H}$. from cochain to chain complexes sending a cochain complex $C^{\bullet}=\left(C^{k}, d^{k}\right)_{k \in \mathbb{Z}}$ to the chain complex $C^{-\bullet}=C_{\bullet}=\left(C_{k}, d_{k}\right)_{k \in \mathbb{Z}}$ given by $C_{k}=C^{-k}, d_{k}=d^{-k}$ allows us to pass freely between cochain and chain complexes. For instance, the identities

$$
\operatorname{Hom}^{-\bullet}\left(C^{\bullet}, D^{\bullet}\right)=\operatorname{Hom}_{\bullet}\left(C^{-\bullet}, D^{-\bullet}\right)
$$

and

$$
\left(C^{\bullet}[n]\right)^{-\bullet}=C^{-\bullet}[-n]
$$

hold.

Let $X^{n}$ be an $n$-dimensional, topological pseudomanifold, equipped with a base point and a CW structure. For a perversity $\bar{p}$, let $I C \bar{\bullet}(X ; \mathbb{Q})$ denote the corresponding intersection chain complex of $X$ with rational coefficients. In fact, let us be more precise about the incarnation of $I C \overline{\boldsymbol{\rho}}(X ; \mathbb{Q})$ that we work with. For a locally compact space $Y$ of finite cohomological dimension, let $\mathcal{D}^{b}(Y)$ denote the bounded derived category of differential complexes of sheaves of rational vector spaces on $Y$, that is, the homotopy category of bounded cochain complexes of sheaves over $\mathbb{Q}$ localized at the quasi-isomorphisms. Let $\mathbf{I C}_{\bar{p}}^{\bullet}(X) \in \mathcal{D}^{b}(X)$ be the intersection sheaf complex on $X$, constructed via Deligne's formula starting from the shifted orientation sheaf on the top stratum, with respect to any topological stratification of $X$ (for example, the intrinsic filtration). The map $f: X \rightarrow$ pt to a point induces a derived pushforward $R f_{*}: \mathcal{D}^{b}(X) \rightarrow \mathcal{D}^{b}(\mathrm{pt})$. Then $R f_{*} \mathbf{I C}_{\bar{p}}^{\bullet}(X)$ is a cochain complex and we set

$$
I C_{\bar{p}}^{\bullet}(X ; \mathbb{Q})=R f_{*} \mathbf{I C}_{\bar{p}}^{\bullet}(X) .
$$

The incarnation of the perversity $\bar{p}$ intersection chain complex that we work with is

$$
I C_{\bullet}^{\bar{p}}(X ; \mathbb{Q})=I C_{\bar{p}}^{-\bullet}(X ; \mathbb{Q}) .
$$

Corollary 2.2 strongly suggests that we define the rational intersection $E$-homology $I E_{k}^{\bar{p}}(X ; \mathbb{Q})$ of $X$ for an $S$-module $E$ as

$$
I E_{k}^{\bar{p}}(X ; \mathbb{Q})=E_{k}\left(\Phi I C_{\bullet}^{\bar{p}}(X ; \mathbb{Q})\right) .
$$

This is in fact fine; it turns out, however, that in the framework of $H \mathbb{Q}$-modules it is technically advantageous to proceed as follows. Let $R$ be a commutative $S$-algebra 
and $E$ an $R$-module. Define the $E$-homology over $R$ and $E$-cohomology over $R$ of an $R$-module $N$ to be

$$
E_{k}^{R}(N)=\pi_{k}\left(E \wedge_{R} N\right), \quad E_{R}^{k}(N)=\pi_{-k}\left(F_{R}(N, E)\right),
$$

where $F_{R}(N, E)$ denotes the function $R$-module. Now let $E$ be an $S$-module. Then $E \mathbb{Q}=E \wedge_{S} H \mathbb{Q}$ is an $H \mathbb{Q}$-module.

Proposition 2.3. For an $S$-module $E$ and $H \mathbb{Q}$-module $N$,

$$
(E \mathbb{Q})_{k}^{H \mathbb{Q}}(N) \cong E_{k}(N) .
$$

Proof. Using the natural isomorphism

$$
\left(E \wedge_{S} H \mathbb{Q}\right) \wedge_{H \mathbb{Q}} N \cong E \wedge_{S} N
$$

of $H \mathbb{Q}$-modules, we have

$$
\begin{aligned}
E_{k}(N) & =\pi_{k}\left(E \wedge_{S} N\right) \\
& \cong \pi_{k}\left(\left(E \wedge_{S} H \mathbb{Q}\right) \wedge_{H \mathbb{Q}} N\right) \\
& =\pi_{k}\left(E \mathbb{Q} \wedge_{H \mathbb{Q}} N\right) \\
& =(E \mathbb{Q})_{k}^{H \mathbb{Q}}(N) .
\end{aligned}
$$

Taking $N=\Phi I C \bar{\bullet}(X ; \mathbb{Q})$, we obtain:

Corollary 2.4. For an $S$-module $E$,

$$
E_{k}\left(\Phi I C_{\bullet}^{\bar{p}}(X ; \mathbb{Q})\right) \cong(E \mathbb{Q})_{k}^{H \mathbb{Q}}\left(\Phi I C_{\bullet}^{\bar{\bullet}}(X ; \mathbb{Q})\right) .
$$

Guided by this corollary and Corollary 2.2, we thus adopt the following definition:

Definition 2.5. Let $E$ be any $S$-module. The rational perversity $\bar{p}$ intersection $E$ homology $I E_{k}^{\bar{p}}(X ; \mathbb{Q})$ of $X$ is defined to be

$$
I E_{k}^{\bar{p}}(X ; \mathbb{Q})=(E \mathbb{Q})_{k}^{H \mathbb{Q}}\left(\Phi I C_{\bullet}^{\bar{p}}(X ; \mathbb{Q})\right) .
$$

The rational perversity $\bar{p}$ intersection $E$-cohomology $I E_{\bar{p}}^{k}(X ; \mathbb{Q})$ of $X$ is defined to be

$$
I E_{\bar{p}}^{k}(X ; \mathbb{Q})=(E \mathbb{Q})_{H \mathbb{Q}}^{k}\left(\Phi I C_{\bullet}^{\bar{p}}(X ; \mathbb{Q})\right) .
$$

Remark 2.6. Because the Hurewicz map $h: S \rightarrow H \mathbb{Z}$ is not a weak equivalence, trying to set

$$
I E_{k}^{\bar{p}}(X ; \mathbb{Z})=(E \mathbb{Z})_{k}^{H \mathbb{Z}}\left(\Phi I C_{\bullet}^{\bar{p}}(X ; \mathbb{Z})\right)
$$

leads, of course, to an incorrect definition of $I E_{k}^{\bar{p}}(X ; \mathbb{Z})$. This can be seen by taking, for instance, $E=S$, the sphere spectrum, and $X$ a point. Then

$$
I E_{*}^{\bar{p}}(X ; \mathbb{Z})=E_{*}(X)=\pi_{*}(S),
$$

the stable homotopy groups of spheres, whereas

$$
\begin{aligned}
(E \mathbb{Z})_{*}^{H \mathbb{Z}}\left(\Phi I C_{\bullet}^{\bar{p}}(X ; \mathbb{Z})\right) & =\pi_{*}\left(\left(E \wedge_{S} H \mathbb{Z}\right) \wedge_{H \mathbb{Z}} \Phi I C_{\bullet}^{\bar{p}}(X ; \mathbb{Z})\right) \\
& \cong \pi_{*}\left(\Phi I C_{\bullet}^{\bar{p}}(X ; \mathbb{Z})\right) \\
& \cong H_{*}\left(I C_{\bullet}^{\bar{p}}(X ; \mathbb{Z})\right) \quad(\text { by }(4)) \\
& \cong H_{*}(\mathrm{pt} ; \mathbb{Z}) .
\end{aligned}
$$


Remark 2.7. Other variants of intersection $E$-(co)homology can easily be defined. For example, we define intersection $E$-homology with compact supports, $I E_{k}^{c, \bar{p}}(X ; \mathbb{Q})$ by $I E_{k}^{c, \bar{p}}(X ; \mathbb{Q})=(E \mathbb{Q})_{k}^{H \mathbb{Q}}\left(\Phi I C_{\bullet}^{c, \bar{p}}(X ; \mathbb{Q})\right)$, where $I C_{\bullet}^{c, \bar{p}}(X ; \mathbb{Q})$ denotes intersection chains with compact support, i.e., the corresponding cochain complex is given by $R f_{!} \mathbf{I C}_{\bar{p}}^{\bullet}(X), f: X \rightarrow$ pt. As another example, if $\mathcal{S}$ is a local coefficient system on the top stratum, then we define intersection $E$-homology with coefficients in $\mathcal{S}$, $I E_{k}^{\bar{p}}(X ; \mathcal{S})$ by $I E_{k}^{\bar{p}}(X ; \mathcal{S})=(E \mathbb{Q})_{k}^{H \mathbb{Q}}\left(\Phi I C_{\bullet}^{\bar{p}}(X ; \mathcal{S})\right)$, where $I C_{\bullet}^{\bar{p}}(X ; \mathcal{S})$ denotes intersection chains with coefficients in $\mathcal{S}$, i.e., the corresponding cochain complex is given by $R f_{*} \mathbf{I C}_{\bar{p}}^{\bullet}(X ; \mathcal{S}), f: X \rightarrow$ pt. To construct $\mathbf{I C}_{\bar{p}}^{\bullet}(X ; \mathcal{S})$ via the Deligne process, one simply starts with $\mathcal{S}$, instead of the orientation sheaf, on the top stratum.

The following lemma shows what we do if $E$ is not an $S$-module but only a CW spectrum: We replace $E$ by the free $S$-module $\mathbb{F}_{S} E$. This does not change the homotopy type:

Lemma 2.8. If $E$ is a $C W$ spectrum, then there is a homotopy equivalence of spectra

$$
E \simeq \mathbb{F}_{S} E
$$

Proof. The natural map

$$
\mathbb{F}_{S} E=S \wedge_{\mathcal{L}} \mathbb{L} E \longrightarrow \mathbb{L} E
$$

is a homotopy equivalence of spectra if $E$ is $\mathrm{CW}$. (Here, $\mathbb{L} E$ denotes the free $\mathbb{L}_{-}$ spectrum generated by $E$.) The unit

$$
E \longrightarrow \mathbb{L} E
$$

is a homotopy equivalence, provided $E$ is $\mathrm{CW}$.

The results of this paper are stated mostly for $E$ an $S$-module. However, the lemma shows that we may replace " $S$-module" by "CW spectrum" and the results continue to hold with $E$ replaced by $\mathbb{F}_{S} E$.

\section{Fundamental Consistency Properties}

Proposition 3.1. If $E=H \mathbb{Z}$ is an Eilenberg-MacLane spectrum $K(\mathbb{Z}, 0)$, then

$$
I E_{k}^{\bar{p}}(X ; \mathbb{Q}) \cong I H_{k}^{\bar{p}}(X ; \mathbb{Q}),
$$

where $I H_{*}^{\bar{p}}(X ; \mathbb{Q})$ denotes the intersection homology groups as defined in [GM83].

Proof. The Hurewicz map $h: S \rightarrow H \mathbb{Z}$ induces a map

$$
H \mathbb{Q} \cong S \wedge_{S} H \mathbb{Q} \stackrel{h \wedge_{S} 1}{\longrightarrow} H \mathbb{Z} \wedge_{S} H \mathbb{Q},
$$

which is an equivalence by Serre's theorem. Therefore,

$$
\begin{aligned}
I(H \mathbb{Z})_{k}^{\bar{p}}(X ; \mathbb{Q}) & =\pi_{k}\left(\left(H \mathbb{Z} \wedge_{S} H \mathbb{Q}\right) \wedge_{H \mathbb{Q}} \Phi I C_{\bullet}^{\bar{p}}(X ; \mathbb{Q})\right) \\
& \cong \pi_{k}\left(H \mathbb{Q} \wedge_{H \mathbb{Q}} \Phi I C_{\bullet}^{\bar{p}}(X ; \mathbb{Q})\right) \\
& \cong \pi_{k}\left(\Phi I C_{\bullet}^{\bar{p}}(X ; \mathbb{Q})\right) \\
& \cong H_{k}\left(I C_{\bullet}^{\bar{p}}(X ; \mathbb{Q})\right) \\
& =I H_{k}^{\bar{p}}(X ; \mathbb{Q}) .
\end{aligned}
$$


Proposition 3.2. If $X$ is a compact $P L$ n-manifold, then

$$
I E_{k}^{\bar{p}}(X ; \mathbb{Q}) \cong E_{k}(X) \otimes \mathbb{Q} .
$$

Proof. For an unoriented manifold $X, \mathbf{I C}_{\bar{p}}^{\bullet}(X)=\mathcal{O}_{X}[n]$, where $\mathcal{O}_{X}$ is the orientation sheaf, and thus

$$
I C_{\bar{p}}^{\bullet}(X ; \mathbb{Q})=R f_{*} \mathcal{O}_{X}[n]=R \Gamma\left(X ; \mathcal{O}_{X}[n]\right) .
$$

Let $U \subset X$ be an open subset. For an admissible (locally finite) triangulation $T$ of $U$, let $C_{\bullet}^{T}(U)$ denote the complex of (possibly infinite) rational simplicial chains of $T$. If $T^{\prime}$ is a subdivision of $T$, then there is a natural morphism of complexes $C_{\bullet}^{T}(U) \rightarrow C_{\bullet}^{T^{\prime}}(U)$ and the PL chain complex of $U$ is defined as the direct limit

$$
C_{\bullet}^{P L}(U)=\lim _{T} C_{\bullet}^{T}(U) .
$$

The presheaf

$$
U \mapsto C_{k}^{P L}(U)
$$

on $X$ is in fact a sheaf and thus defines a sheaf complex $\mathbf{C}^{\bullet}$ with

$$
\Gamma\left(U ; \mathbf{C}^{-k}\right)=C_{k}^{P L}(U) .
$$

This sheaf complex is fine, since each $\mathbf{C}^{-k}$ is a module over the sheaf of constructible functions, that is, functions constant on interiors of simplices, which is fine. The right derived functor of the section functor may be computed by means of a fine resolution. Hence, $R \Gamma\left(X ; \mathbf{C}^{\bullet}\right)=\Gamma\left(X ; \mathbf{C}^{\bullet}\right)$. The dualizing complex $\mathbb{D}_{X}^{\bullet}$ on $X$ is quasi-isomorphic to $\mathbf{C}^{\bullet}$. Since $X$ is a manifold, $\mathbb{D}_{X}^{\bullet}$ is further canonically quasi-isomorphic to the shifted orientation sheaf $\mathcal{O}_{X}[n]$. Summarizing,

$$
\mathcal{O}_{X}[n] \cong \mathbf{C}^{\bullet}
$$

and consequently,

$$
R \Gamma\left(X ; \mathcal{O}_{X}[n]\right) \cong R \Gamma\left(X ; \mathbf{C}^{\bullet}\right)=\Gamma\left(X ; \mathbf{C}^{\bullet}\right)=C_{-}^{P L}(X)
$$

in $\mathcal{D}^{b}(\mathrm{pt})$. Let $T$ be any admissible triangulation of $X$ and let $S \bullet(X)$ denote the rational singular chain complex of $X$. If $X$ is compact, there is an obvious chain map

$$
C_{\bullet}^{T}(X) \longrightarrow S_{\bullet}(X)
$$

which is a quasi-isomorphism. The canonical chain map

$$
C_{\bullet}^{T}(X) \longrightarrow C_{\bullet}^{P L}(X)
$$

is a quasi-isomorphism as well, and so the roof

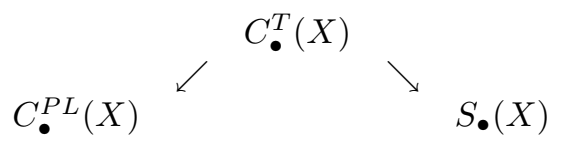

defines an isomorphism $C_{\bullet}^{P L}(X) \cong S_{\bullet}(X)$ in $\mathcal{D}(\mathbb{Q})$. The triangulation $T$ defines a CW structure on $X$ with skeleta $\left\{X^{p}\right\}$. Following [Wal65], the filtration $\left\{X^{p}\right\}$ of $X$ 
induces a filtration $F^{p} S_{\bullet}(X)$ of $S_{\bullet}(X)$ by

$$
F^{p} S_{\bullet}(X)=\operatorname{Im}\left(S_{\bullet}\left(X^{p}\right) \rightarrow S_{\bullet}(X)\right) .
$$

Define a subcomplex $D_{\bullet}(X)$ of $S_{\bullet}(X)$ by

$$
D_{p}(X)=\operatorname{ker}\left(\partial: F^{p} S_{p}(X) \longrightarrow F^{p} S_{p-1}(X) / F^{p-1} S_{p-1}(X)\right) .
$$

Then the cellular chain complex $C_{\bullet}(X ; \mathbb{Q})$ is a quotient complex of $D_{\bullet}(X)$. By

[Wal65, Lemma 1], the natural chain maps

$$
S_{\bullet}(X) \hookleftarrow D_{\bullet}(X) \rightarrow C_{\bullet}(X ; \mathbb{Q})
$$

are chain equivalences. We conclude

$$
I C_{\bullet}^{\bar{p}}(X ; \mathbb{Q}) \cong C_{\bullet}^{P L}(X) \cong S_{\bullet}(X) \cong C_{\bullet}(X ; \mathbb{Q})
$$

in $\mathcal{D}(\mathbb{Q})$. The statement follows from applying $\Phi$ followed by $(E \mathbb{Q})_{*}^{H \mathbb{Q}}$, then using Proposition 2.3 with $N=\Phi C \bullet(X ; \mathbb{Q})$, and applying Corollary 2.2 .

\section{Topological Invariance}

Proposition 4.1. Let $X$ and $Y$ be n-dimensional topological pseudomanifolds. Then any homeomorphism $f: X \rightarrow Y$ induces isomorphisms

$$
I E_{*}^{\bar{p}}(X ; \mathbb{Q}) \cong I E_{*}^{\bar{p}}(Y ; \mathbb{Q}), \quad I E_{\bar{p}}^{*}(X ; \mathbb{Q}) \cong I E_{\bar{p}}^{*}(Y ; \mathbb{Q})
$$

for any $S$-module $E$.

Proof. By [GM83],

$$
\mathbf{I C}_{\bar{p}}^{\bullet}(Y) \cong R f_{*} \mathbf{I C}_{\bar{p}}^{\bullet}(X)
$$

in $\mathcal{D}^{b}(Y)$. If $g: Y \rightarrow$ pt is the map to a point, then

$$
I C_{\bar{p}}^{\bullet}(Y ; \mathbb{Q})=R g_{*} \mathbf{I C}_{\bar{p}}^{\bullet}(Y) \cong R g_{*} R f_{*} \mathbf{I C}_{\bar{p}}^{\bullet}(X) \cong R(g f)_{*} \mathbf{I C}_{\bar{p}}^{\bullet}(X)=I C_{\bar{p}}^{\bullet}(X ; \mathbb{Q}) .
$$

Passing to chain complexes and applying $\Phi$ yields an isomorphism

$$
\Phi I C_{\bullet}^{\bar{p}}(Y ; \mathbb{Q}) \cong \Phi I C_{\bullet}^{\bar{p}}(X ; \mathbb{Q})
$$

in $\mathcal{D}(H \mathbb{Q})$. Smashing with $E \mathbb{Q}$ preserves weak equivalences:

$$
\Phi I C_{\bullet}^{\bar{p}}(Y ; \mathbb{Q}) \wedge_{H \mathbb{Q}} E \mathbb{Q} \cong \Phi I C_{\bullet}^{\bar{p}}(X ; \mathbb{Q}) \wedge_{H \mathbb{Q}} E \mathbb{Q},
$$

so that on homotopy groups

$$
I E_{k}^{\bar{p}}(Y ; \mathbb{Q})=\pi_{k}\left(\Phi I C_{\bullet}^{\bar{p}}(Y ; \mathbb{Q}) \wedge_{H \mathbb{Q}} E \mathbb{Q}\right) \cong \pi_{k}\left(\Phi I C_{\bullet}^{\bar{p}}(X ; \mathbb{Q}) \wedge_{H \mathbb{Q}} E \mathbb{Q}\right)=I E_{k}^{\bar{p}}(X ; \mathbb{Q}) .
$$

Similarly, for cohomology, (8) induces

$$
F_{H \mathbb{Q}}\left(\Phi I C_{\bullet}^{\bar{p}}(Y ; \mathbb{Q}), E \mathbb{Q}\right) \cong F_{H \mathbb{Q}}\left(\Phi I C_{\bullet}^{\bar{p}}(X ; \mathbb{Q}), E \mathbb{Q}\right),
$$

which on homotopy groups induces the desired isomorphism between cohomology groups. 


\section{Generalized Poincaré Duality}

We emphasize that in the following lemma, the chain complex $C_{\bullet}$ is not assumed to have bounded homology.

Lemma 5.1. Let $A_{\bullet}, B_{\bullet}$ and $C_{\bullet}$ be rational chain complexes such that $A_{\bullet}$ and $B_{\bullet}$ have nonzero homology only in a bounded range and $A_{\bullet}$ has finite dimensional homology in every degree. Then the natural map

$$
\operatorname{Hom}_{\bullet}\left(A_{\bullet}, B_{\bullet}\right) \otimes C_{\bullet} \longrightarrow \operatorname{Hom}_{\bullet}\left(A_{\bullet}, B_{\bullet} \otimes C_{\bullet}\right)
$$

is an isomorphism in $\mathcal{D}(\mathbb{Q})$.

For $B_{\bullet}=\mathbb{Q}[0]$, the lemma asserts

$$
\operatorname{Hom}_{\bullet}\left(A_{\bullet}, \mathbb{Q}[0]\right) \otimes C_{\bullet} \cong \operatorname{Hom}_{\bullet}\left(A_{\bullet}, C_{\bullet}\right),
$$

which may be interpreted via $\Phi$ as algebraic Spanier-Whitehead duality for $H \mathbb{Q}$ modules: Recall that if $R$ is a commutative $S$-algebra and $M$ is an $R$-module, then the Spanier-Whitehead dual $D_{R} M$ of $M$ is defined to be the function $R$-module $D_{R} M=F_{R}(M, R)$. The functor $\Phi$ converts homomorphism modules into function modules,

$$
\Phi \operatorname{Hom}_{\mathbb{Q}}\left(C_{\bullet}, D_{\bullet}\right) \cong F_{H \mathbb{Q}}\left(\Phi C_{\bullet}, \Phi D_{\bullet}\right)
$$

in $\mathcal{D}(H \mathbb{Q})$.

Proposition 5.2 (Spanier-Whitehead Duality for $H \mathbb{Q}$-modules). Let $E$ be an $S$ module and $M$ an $H \mathbb{Q}$-module having nonzero homotopy groups only in a bounded range and finite dimensional homotopy $\mathbb{Q}$-vector spaces in every degree. Then there is an isomorphism

$$
(E \mathbb{Q})_{k}^{H \mathbb{Q}}\left(D_{H \mathbb{Q}} M\right) \cong(E \mathbb{Q})_{H \mathbb{Q}}^{-k}(M) .
$$

Proof. Set $A_{\bullet}=C h(M), C_{\bullet}=C h(E \mathbb{Q})$. Then $H_{k}\left(A_{\bullet}\right) \cong \pi_{k}(M)$ and $A_{\bullet}$ has bounded homology with finite dimensional homology groups in every degree. Thus, (9) applies and yields

$$
\operatorname{Hom}_{\bullet}(C h(M), \mathbb{Q}[0]) \otimes C h(E \mathbb{Q}) \cong \operatorname{Hom}_{\bullet}(C h(M), C h(E \mathbb{Q})) .
$$

Applying the functor $\Phi$ to this isomorphism and using (3), (10) with $R=\mathbb{Q}$, we obtain

$$
F_{H \mathbb{Q}}(\Phi C h(M), \Phi \mathbb{Q}[0]) \wedge_{H \mathbb{Q}} \Phi C h(E \mathbb{Q}) \cong F_{H \mathbb{Q}}(\Phi C h(M), \Phi C h(E \mathbb{Q})) .
$$

Using

$$
\Phi \mathbb{Q}[0]=H \mathbb{Q}, \quad \Phi C h(M) \cong M, \quad \Phi C h(E \mathbb{Q}) \cong E \mathbb{Q},
$$

this can be rewritten as

$$
D_{H \mathbb{Q}}(M) \wedge_{H \mathbb{Q}} E \mathbb{Q} \cong F_{H \mathbb{Q}}(M, E \mathbb{Q}),
$$

which induces on homotopy groups

$$
(E \mathbb{Q})_{k}^{H \mathbb{Q}}\left(D_{H \mathbb{Q}} M\right)=\pi_{k}\left(D_{H \mathbb{Q}}(M) \wedge_{H \mathbb{Q}} E \mathbb{Q}\right) \cong \pi_{k}\left(F_{H \mathbb{Q}}(M, E \mathbb{Q})\right)=(E \mathbb{Q})_{H \mathbb{Q}}^{-k}(M) .
$$


Given a perversity $\bar{p}$, let $\bar{q}$ denote its complementary perversity, that is, $\bar{p}+\bar{q}=\bar{t}$, where $\bar{t}$ is the top perversity. An orientation of $X$ is an isomorphism $\mathcal{O}_{X-\Sigma} \cong \mathbb{R}_{X-\Sigma}$, where $X-\Sigma$ is the top stratum of $X$.

Theorem 5.3. For compact, oriented $X$, there is a generalized Poincaré duality isomorphism

$$
I E_{n-k}^{\bar{q}}(X ; \mathbb{Q}) \cong I E_{\bar{p}}^{k}(X ; \mathbb{Q})
$$

for any $S$-module $E$.

Proof. For a locally compact space $Y$ of finite cohomological dimension, $D_{Y}: \mathcal{D}^{b}(Y)$ $\rightarrow \mathcal{D}^{b}(Y)$ denotes the Verdier dualizing functor defined by

$$
D_{Y}=R \operatorname{Hom}^{\bullet}\left(-, \mathbb{D}_{Y}^{\bullet}\right),
$$

where $\mathbb{D}_{Y}^{\bullet}$ is the Verdier dualizing complex of $Y$. Generalized Poincaré duality between intersection homology groups is induced, using the orientation of $X$, by a sheaf level isomorphism

$$
d: D_{X} \mathbf{I C}_{\bar{p}}^{\bullet}(X)[n] \stackrel{\simeq}{\longrightarrow} \mathbf{I C}_{\bar{q}}^{\bullet}(X)
$$

in $\mathcal{D}^{b}(X)$. As $X$ was assumed to be compact, $f: X \rightarrow$ pt is a proper map and thus $R f_{*}=R f_{!}$. Therefore, $d$ induces

$$
\begin{aligned}
D_{\mathrm{pt}} I C_{\bar{p}}^{\bullet}(X ; \mathbb{Q})[n] & =D_{\mathrm{pt}} R f_{*} \mathbf{I} \mathbf{C}_{\bar{p}}^{\bullet}(X)[n] \\
& \cong R f_{!} D_{X} \mathbf{I} \mathbf{C}_{\bar{p}}^{\bullet}(X)[n] \\
& =R f_{*} D_{X} \mathbf{I} \mathbf{C}_{\bar{p}}^{\bullet}(X)[n] \\
& \cong R f_{*} \mathbf{I} \mathbf{C}_{\bar{q}}^{\bullet}(X) \\
& =I C_{\bar{q}}^{\bullet}(X ; \mathbb{Q}) .
\end{aligned}
$$

Let us briefly write $I C_{\bar{p}}^{\bullet}=I C_{\bar{p}}^{\bullet}(X ; \mathbb{Q}), I C_{\bar{q}}^{\bullet}=I C_{\bar{q}}^{\bullet}(X ; \mathbb{Q})$. The dualizing complex of a point is $\mathbb{D}_{\mathrm{pt}}^{\bullet}=\mathbb{Q}[0]$, whence the dualizing functor $D_{\mathrm{pt}}$ can be computed as

$$
D_{\mathrm{pt}}=R \operatorname{Hom}^{\bullet}\left(-, \mathbb{D}_{\mathrm{pt}}^{\bullet}\right)=R \operatorname{Hom}^{\bullet}(-, \mathbb{Q}[0])=\operatorname{Hom}^{\bullet}(-, \mathbb{Q}[0]),
$$

observing that over a point every complex of $\mathbb{Q}$-vector spaces is already injective. Next, let us carry out the passage from $\mathcal{D}^{b}(\mathrm{pt})$ to $\mathcal{D}(\mathbb{Q})$ : The isomorphism in $\mathcal{D}^{b}(\mathrm{pt})$

$$
\operatorname{Hom}^{\bullet}\left(I C_{\bar{p}}^{\bullet}, \mathbb{Q}[0]\right) \cong I C_{\bar{q}}^{\bullet}[-n]
$$

translates to

$$
\operatorname{Hom}_{\bullet}\left(I C_{\bullet}^{\bar{p}}, \mathbb{Q}[0]\right) \cong I C_{\bullet}^{\bar{q}}[n]
$$

in $\mathcal{D}(\mathbb{Q})$, using $(6)$ and $(7)$. By (9) with $A_{\bullet}=I C_{\bullet}^{\bar{p}}, C_{\bullet}=C h(E \mathbb{Q})$, there is an isomorphism

$$
\operatorname{Hom}_{\bullet}\left(I C_{\bullet}^{\bar{p}}, \mathbb{Q}[0]\right) \otimes C h(E \mathbb{Q}) \cong \operatorname{Hom}_{\bullet}\left(I C_{\bullet}^{\bar{p}}, C h(E \mathbb{Q})\right)
$$


The desired duality isomorphism is given by

$$
\begin{array}{rlrl}
I E_{\bar{p}}^{k}(X ; \mathbb{Q}) & =\pi_{-k}\left(F_{H \mathbb{Q}}\left(\Phi I C_{\bullet}^{\bar{p}}, E \mathbb{Q}\right)\right) & \\
& \cong \pi_{-k}\left(F_{H \mathbb{Q}}\left(\Phi I C_{\bullet}^{\bar{p}}, \Phi C h(E \mathbb{Q})\right)\right) & \\
& \cong \pi_{-k}\left(\Phi \operatorname{Hom}_{\bullet}\left(I C^{\overline{\boldsymbol{p}}}, C h(E \mathbb{Q})\right)\right) & & \text { by }(10) \\
& \cong H_{-k}\left(\operatorname{Hom}_{\bullet}\left(I C_{\bullet}^{\bar{p}}, C h(E \mathbb{Q})\right)\right) & & \text { by }(4) \\
& \cong H_{-k}\left(\operatorname{Hom}_{\bullet}\left(I C_{\bullet}^{\bar{p}}, \mathbb{Q}[0]\right) \otimes C h(E \mathbb{Q})\right) & & \text { by }(13) \\
& \cong H_{-k}\left(I C_{\bullet}^{\bar{q}}[n] \otimes C h(E \mathbb{Q})\right) & & \text { by }(12) \\
& \cong H_{n-k}\left(I C_{\bullet}^{\bar{q}} \otimes C h(E \mathbb{Q})\right) & \\
& \cong \pi_{n-k}\left(\Phi\left(I C_{\bullet}^{\bar{q}} \otimes C h(E \mathbb{Q})\right)\right) & \\
& \cong \pi_{n-k}\left(\Phi I C_{\bullet}^{\bar{q}} \wedge H \mathbb{Q} E \mathbb{Q}\right) & \text { by }(4) \\
& =I E_{n-k}^{\bar{q}}(X ; \mathbb{Q}) . & & \text { by }(3)
\end{array}
$$

\section{Small Resolutions of Singularities}

Let $X$ and $Y$ be irreducible, complex, $n$-dimensional, compact, algebraic varieties and let $f: Y \rightarrow X$ be a resolution of singularities. The map $f$ is called small if for all $r>0$,

$$
\operatorname{codim}_{\mathbb{C}}\left\{x \in X \mid \operatorname{dim}_{\mathbb{C}} f^{-1}(x) \geqslant r\right\}>2 r
$$

see [GM83, §6.2]. If $X$ is one or two-dimensional, then a small $f$ must be a finite map. If $X$ is 3 -dimensional, then the fibers of a small $f$ must be 0 -dimensional except possibly over a set of isolated points in $X$, where the fibers may be at most curves. Let $\bar{m}$ denote the middle perversity.

Example 6.1. Consider $\mathbb{C}^{3}$ as a linear subspace of $\mathbb{C}^{5}$ via $\mathbb{C}^{3}=\left\{\left(z_{1}, z_{2}, z_{3}, 0,0\right) \in \mathbb{C}^{5} \mid\right.$ $\left.z_{1}, z_{2}, z_{3} \in \mathbb{C}\right\}$. Let $X$ be the singular Schubert variety

$$
X=\left\{V^{2} \in G_{2}\left(\mathbb{C}^{5}\right) \mid \operatorname{dim}_{\mathbb{C}}\left(V^{2} \cap \mathbb{C}^{3}\right) \geqslant 1\right\},
$$

where $G_{2}\left(\mathbb{C}^{5}\right)$ denotes the Grassmannian of 2-planes in $\mathbb{C}^{5}$. A small resolution of $X$ is given by taking $Y$ to be the variety of partial flags $V^{1} \subset V^{2} \subset \mathbb{C}^{5}$ such that $V^{1} \subset \mathbb{C}^{2}$. The map $f: Y \rightarrow X$ sends the partial flag to $V^{2}$.

Theorem 6.2. If $f: Y \rightarrow X$ is a small resolution of singularities, then

$$
I E_{k}^{\bar{m}}(X ; \mathbb{Q}) \cong E_{k}(Y) \otimes \mathbb{Q} .
$$

Proof. By [GM83, §6.2],

$$
R f_{*} \mathbf{I C}_{\bar{m}}^{\bullet}(Y) \cong \mathbf{I C}_{\bar{m}}^{\bullet}(X)
$$

in $\mathcal{D}^{b}(X)$. Applying $R g_{*}$ for $g: X \rightarrow$ pt, we obtain

$$
I C_{\bar{m}}^{\bullet}(Y ; \mathbb{Q})=R(g f)_{*} \mathbf{I C}_{\bar{m}}^{\bullet}(Y) \cong R g_{*} \mathbf{I C}_{\bar{m}}^{\bullet}(X)=I C_{\bar{m}}^{\bullet}(X ; \mathbb{Q})
$$

in $\mathcal{D}^{b}(\mathrm{pt})$. Converting to chain complexes, and applying $\Phi$ followed by $(E \mathbb{Q})_{k}^{H \mathbb{Q}}$, induces an isomorphism

$$
I E_{k}^{\bar{m}}(Y ; \mathbb{Q})=(E \mathbb{Q})_{k}^{H \mathbb{Q}}\left(\Phi I C_{\bullet}^{\bar{m}}(Y ; \mathbb{Q})\right) \cong(E \mathbb{Q})_{k}^{H \mathbb{Q}}\left(\Phi I C_{\bullet}^{\bar{m}}(X ; \mathbb{Q})\right)=I E_{k}^{\bar{m}}(X ; \mathbb{Q})
$$

As $Y$ is a manifold, it follows from Proposition 3.2 that $I E_{k}^{\bar{m}}(Y ; \mathbb{Q}) \cong E_{k}(Y) \otimes \mathbb{Q}$. 
Corollary 6.3. Let $E$ be any $S$-module. If $Y$ and $Y^{\prime}$ are two small resolutions of $X$, then

$$
E_{k}(Y) \otimes \mathbb{Q} \cong E_{k}\left(Y^{\prime}\right) \otimes \mathbb{Q} .
$$

Remark 6.4. If $E$ is an $S$-algebra, then the ring structures are in general not preserved by the above isomorphism. In fact, the rings need not even be abstractly isomorphic. Examples can already be obtained for $E$ the Eilenberg-MacLane spectrum $H \mathbb{Z}$.

Example 6.5. We continue the previous Example 6.1. The variety $Y^{\prime}$ of partial flags $V^{2} \subset V^{4} \subset \mathbb{C}^{5}$ such that $\mathbb{C}^{3} \subset V^{4}$ is another small resolution of $X$. The cohomology rings $H^{*}(Y)$ and $H^{*}\left(Y^{\prime}\right)$ are not isomorphic; see [B $\mathbf{B}^{+} \mathbf{8 4}$, IX].

\section{Products and Operations}

Let $R$ be a commutative $S$-algebra and let $E, M$ and $N$ be $R$-modules. Consider the natural map

$$
F_{R}(M, E) \wedge_{R} F_{R}(N, E) \longrightarrow F_{R}\left(M \wedge_{R} N, E \wedge_{R} E\right) .
$$

On homotopy groups, the composition

$$
\pi_{*}\left(F_{R}(M, E)\right) \otimes_{\pi_{*} R} \pi_{*}\left(F_{R}(N, E)\right) \longrightarrow \pi_{*}\left(F_{R}(M, E) \wedge_{R} F_{R}(N, E)\right)
$$

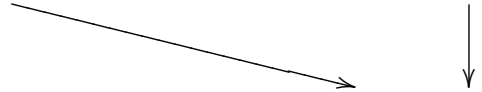

defines an external cohomology product

$$
E_{R}^{*}(M) \otimes_{R_{*}} E_{R}^{*}(N) \longrightarrow\left(E \wedge_{R} E\right)_{R}^{*}\left(M \wedge_{R} N\right) .
$$

Now let $\bar{p}$ be a perversity such that

$$
\bar{p}(k)+\bar{p}(l) \leqslant \bar{p}(k+l) \leqslant \bar{p}(k)+\bar{p}(l)+2
$$

for all $k, l$ (for example, $\bar{p}=\bar{m}$ ), let $E$ be an $S$-algebra, let $X$ and $Y$ be compact pseudomanifolds, and specialize to $R=H \mathbb{Q}, M=\Phi I C_{\bullet}^{\bar{p}}(X ; \mathbb{Q}), N=\Phi I C_{\bullet}^{\bar{p}}(Y ; \mathbb{Q})$. Using the isomorphism

$$
M \wedge_{H \mathbb{Q}} N \cong \Phi\left(I C_{\bullet}^{\bar{p}}(X ; \mathbb{Q}) \otimes I C_{\bullet}^{\bar{p}}(Y ; \mathbb{Q})\right) \cong \Phi I C_{\bullet}^{\bar{p}}(X \times Y ; \mathbb{Q})
$$

(the latter isomorphism is induced by applying $\Phi$ to the Eilenberg-Zilber isomorphism (23) in the proof of the Künneth Theorem 9.1) yields a product

$$
\begin{aligned}
(E \mathbb{Q})_{H \mathbb{Q}}^{*}\left(\Phi I C_{\bullet}^{\bar{\bullet}}(X ; \mathbb{Q})\right) \otimes_{\mathbb{Q}}(E \mathbb{Q})_{H \mathbb{Q}}^{*}\left(\Phi I C_{\bullet}^{\bar{\varphi}}(Y ; \mathbb{Q})\right) & \\
& \longrightarrow\left(E \mathbb{Q} \wedge_{H \mathbb{Q}} E \mathbb{Q}\right)_{H \mathbb{Q}}^{*}\left(\Phi I C_{\bullet}^{\bar{\bullet}}(X \times Y ; \mathbb{Q})\right) .
\end{aligned}
$$

The multiplication $\mu: E \wedge_{S} E \longrightarrow E$ induces a multiplication

$$
\mu \mathbb{Q}: E \mathbb{Q} \wedge_{H \mathbb{Q}} E \mathbb{Q} \longrightarrow E \mathbb{Q}
$$


by

$$
\begin{aligned}
E \mathbb{Q} \wedge_{H \mathbb{Q}} E \mathbb{Q} & =\left(E \wedge_{S} H \mathbb{Q}\right) \wedge_{H \mathbb{Q}}\left(E \wedge_{S} H \mathbb{Q}\right) \\
& \cong E \wedge_{S}\left(E \wedge_{S} H \mathbb{Q}\right) \\
& \cong\left(E \wedge_{S} E\right) \wedge_{S} H \mathbb{Q} \quad \stackrel{\mu \wedge_{S} 1_{H \mathbb{Q}}}{\longrightarrow} \wedge_{S} H \mathbb{Q}=E \mathbb{Q} .
\end{aligned}
$$

Composing the product (15) with the map induced by $\mu \mathbb{Q}$ defines an external intersection $E$-cohomology product

$$
I E_{\bar{p}}^{*}(X ; \mathbb{Q}) \otimes_{\mathbb{Q}} I E_{\bar{p}}^{*}(Y ; \mathbb{Q}) \longrightarrow I E_{\bar{p}}^{*}(X \times Y ; \mathbb{Q}) .
$$

In order to obtain internal products, one must study the diagonal map

$$
\Delta: X \longrightarrow X \times X \text {. }
$$

Here, $X \times X$ is endowed with the product stratification, that is, if

$$
X_{0} \subset X_{1} \subset X_{2} \subset \cdots
$$

are the closed strata of $X$, then

$$
X_{0} \times X_{0} \subset X_{1} \times X_{0} \cup X_{0} \times X_{1} \subset X_{2} \times X_{0} \cup X_{1} \times X_{1} \cup X_{0} \times X_{2} \subset \cdots
$$

are the closed strata of $X \times X$. If $\mathcal{X}$ denotes the collection of components of pure strata of $X$, then

$$
\{S \times T: S, T \in \mathcal{X}\}
$$

are the components of pure strata of $X \times X$.

Proposition 7.1. If $\bar{p}, \bar{q}$ are two perversities such that

$$
\bar{q}(k)+k \leqslant \bar{p}(2 k)
$$

for all $k \geqslant 2$, then the diagonal map $\Delta: X \rightarrow X \times X$ induces a pushforward of intersection chains

$$
\Delta_{*}: I C_{i}^{\bar{q}}(X) \longrightarrow I C_{i}^{\bar{p}}(X \times X) .
$$

Proof. We will argue geometrically using PL chains, leaving the sheaf-theoretic formulation for topological pseudomanifolds to the reader. Let $\xi \in I C_{i}^{\bar{q}}(X)$ be an intersection chain allowable with respect to $\bar{q}$. We will show that

$$
\operatorname{dim}(\Delta|\xi| \cap(S \times T)) \leqslant i-\operatorname{codim}(S \times T)+\bar{p}(\operatorname{codim}(S \times T))
$$

for all $S, T \in \mathcal{X}$. If $S \neq T$, then $S \cap T=\varnothing$ and $\Delta(X) \cap(S \times T)=\varnothing$. Thus, (17) holds in this case and we may now concentrate on the case $S=T$. Since $\Delta: X \rightarrow \Delta(X)$ is a homeomorphism, we have

$$
\begin{gathered}
|\xi| \cong \Delta|\xi|, \\
|\xi| \cap S \cong \Delta(|\xi| \cap S) .
\end{gathered}
$$

As $\Delta$ is injective,

$$
\Delta(|\xi| \cap S)=\Delta|\xi| \cap \Delta(S) .
$$

We also note that

$$
\Delta|\xi| \cap(S \times S)=\Delta|\xi| \cap \Delta(S) .
$$


If $k=\operatorname{codim} S$, then $\operatorname{codim}(S \times S)=2 k$. Using the $\bar{q}$-allowability of $\xi$, the following calculation establishes (17):

$$
\begin{aligned}
\operatorname{dim}(\Delta|\xi| \cap(S \times S)) & =\operatorname{dim}(|\xi| \cap S) \quad \text { (by }(19),(20) \text { and }(21)) \\
& \leqslant i-k+\bar{q}(k) \\
& \leqslant i-2 k+\bar{p}(2 k) \quad(\text { by }(16)) \\
& =i-\operatorname{codim}(S \times S)+\bar{p}(\operatorname{codim}(S \times S))
\end{aligned}
$$

where $i$ is indeed the dimension of $\Delta|\xi|$ by (18). Thus, $\xi \mapsto \Delta(\xi)$ defines the chain $\operatorname{map} \Delta_{*}$.

Applying the functor $\Phi$ to $\Delta_{*}$ yields a morphism

$$
\Phi \Delta_{*}: \Phi I C_{\bullet}^{\bar{q}}(X ; \mathbb{Q}) \longrightarrow \Phi I C_{\bullet}^{\bar{p}}(X \times X ; \mathbb{Q})
$$

which induces a morphism

$$
F_{H \mathbb{Q}}\left(\Phi \Delta_{*}, 1\right): F_{H \mathbb{Q}}\left(\Phi I C_{\bullet}^{\bar{p}}(X \times X ; \mathbb{Q}), E \mathbb{Q}\right) \longrightarrow F_{H \mathbb{Q}}\left(\Phi I C_{\bullet}^{\bar{q}}(X ; \mathbb{Q}), E \mathbb{Q}\right)
$$

on function $H \mathbb{Q}$-modules. On homotopy groups, $\pi_{-*} F_{H \mathbb{Q}}\left(\Phi \Delta_{*}, 1\right)$ defines a map

$$
\Delta^{*}: I E_{\bar{p}}^{*}(X \times X ; \mathbb{Q}) \longrightarrow I E_{\bar{q}}^{*}(X ; \mathbb{Q}) .
$$

Let $(\bar{p}, \bar{q})$ be a pair of perversities such that $\bar{p}$ satisfies the Künneth condition (14) and $(\bar{p}, \bar{q})$ satisfy the diagonal pushforward condition (16). Then the composition of the external product with $\Delta^{*}$ defines an internal (cup) product $\cup$ :

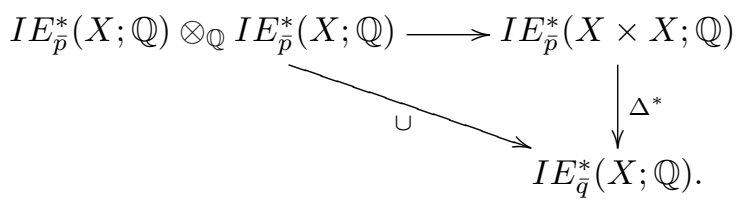

We leave the formulation of other types of products to the reader.

Let us turn our attention to defining operations in $I E$-cohomology of an arbitrary pseudomanifold $X$, for an arbitrary perversity $\bar{p}$. For a commutative $S$-algebra $R$ and $R$-modules $M, N, K$, we start out with the natural, associative and $R$-unital composition pairing

$$
F_{R}(M, N) \wedge_{R} F_{R}(K, M) \longrightarrow F_{R}(K, N) .
$$

Specialization to $R=H \mathbb{Q}, M=N=E \mathbb{Q}$ for any $S$-module $E$ and $K=\Phi I C \bar{\bullet}(X ; \mathbb{Q})$ yields

$$
F_{H \mathbb{Q}}(E \mathbb{Q}, E \mathbb{Q}) \wedge_{H \mathbb{Q}} F_{H \mathbb{Q}}\left(\Phi I C_{\bullet}^{\bar{p}}(X ; \mathbb{Q}), E \mathbb{Q}\right) \longrightarrow F_{H \mathbb{Q}}\left(\Phi I C_{\bullet}^{\bar{p}}(X ; \mathbb{Q}), E \mathbb{Q}\right)
$$

which induces on homotopy groups

$$
(E \mathbb{Q})_{H \mathbb{Q}}^{*}(E \mathbb{Q}) \otimes_{\mathbb{Q}}(E \mathbb{Q})_{H \mathbb{Q}}^{*}\left(\Phi I C_{\bullet}^{\bar{p}}(X ; \mathbb{Q})\right) \longrightarrow(E \mathbb{Q})_{H \mathbb{Q}}^{*}\left(\Phi I C_{\bullet}^{\bar{p}}(X ; \mathbb{Q})\right),
$$

that is,

$$
(E \mathbb{Q})_{H \mathbb{Q}}^{*}(E \mathbb{Q}) \otimes_{\mathbb{Q}} I E_{\bar{p}}^{*}(X ; \mathbb{Q}) \longrightarrow I E_{\bar{p}}^{*}(X ; \mathbb{Q}) .
$$

Thus, every element $\alpha$ of the algebra of operations $(E \mathbb{Q})_{H \mathbb{Q}}^{*}(E \mathbb{Q})$ defines a cohomology 
operation

$$
\alpha: I E_{\bar{p}}^{*}(X ; \mathbb{Q}) \longrightarrow I E_{\bar{p}}^{*}(X ; \mathbb{Q})
$$

\section{Atiyah-Hirzebruch Formulae}

Proposition 8.1. For any $S$-module $E$, there is an isomorphism

$$
I E_{k}^{\bar{p}}(X ; \mathbb{Q}) \cong \bigoplus_{i+j=k} I H_{i}^{\bar{p}}(X ; \mathbb{Q}) \otimes_{\mathbb{Q}}\left(\pi_{j}(E) \otimes_{\mathbb{Z}} \mathbb{Q}\right) .
$$

Proof. This follows readily from the algebraic Künneth theorem:

$$
\begin{aligned}
I E_{k}^{\bar{p}}(X ; \mathbb{Q}) & =\pi_{k}\left(\Phi I C_{\cdot}^{\bar{p}}(X ; \mathbb{Q}) \wedge_{H \mathbb{Q}} E \mathbb{Q}\right) \\
& \cong \pi_{k}\left(\Phi I C_{\bullet}^{\bar{p}}(X ; \mathbb{Q}) \wedge_{H \mathbb{Q}} \Phi C h(E \mathbb{Q})\right) \\
& \cong \pi_{k}\left(\Phi\left(I C_{\bullet}^{\bar{p}}(X ; \mathbb{Q}) \otimes C h(E \mathbb{Q})\right)\right) \\
& \cong H_{k}\left(I C_{\bullet}^{\bar{p}}(X ; \mathbb{Q}) \otimes C h(E \mathbb{Q})\right) \\
& \cong \bigoplus_{i+j=k} H_{i}\left(I C_{\bullet}^{\bar{p}}(X ; \mathbb{Q})\right) \otimes H_{j}(C h(E \mathbb{Q})) \\
& \cong \bigoplus_{i+j=k} I H_{i}^{\bar{p}}(X ; \mathbb{Q}) \otimes \pi_{j}(E \mathbb{Q}) \\
& \cong \bigoplus_{i+j=k} I H_{i}^{\bar{p}}(X ; \mathbb{Q}) \otimes\left(\pi_{j}(E) \otimes \mathbb{Q}\right) .
\end{aligned}
$$

Proposition 8.2. For any $S$-module $E$, there is an isomorphism

$$
I E_{\bar{p}}^{k}(X ; \mathbb{Q}) \cong \prod_{i-j=k} \operatorname{Hom}_{\mathbb{Q}}\left(I H_{i}^{\bar{p}}(X ; \mathbb{Q}), \pi_{j}(E) \otimes_{\mathbb{Z}} \mathbb{Q}\right) .
$$

Proof. Using the cohomological universal coefficient theorem:

$$
\begin{aligned}
I E_{\bar{p}}^{k}(X ; \mathbb{Q}) & =\pi_{-k} F_{H \mathbb{Q}}\left(\Phi I C_{\bullet}^{\bar{p}}(X ; \mathbb{Q}), E \mathbb{Q}\right) \\
& \cong \pi_{-k} F_{H \mathbb{Q}}\left(\Phi I C_{\bullet}^{\bar{p}}(X ; \mathbb{Q}), \Phi C h(E \mathbb{Q})\right) \\
& \cong \pi_{-k} \Phi \operatorname{Hom} \cdot\left(I C_{\bullet}^{\bar{p}}(X ; \mathbb{Q}), C h(E \mathbb{Q})\right) \\
& \cong H_{-k}\left(\operatorname{Hom}_{\bullet}(I C \cdot \bar{p}(X ; \mathbb{Q}), C h(E \mathbb{Q}))\right) \\
& \cong \prod_{i-j=k} \operatorname{Hom}_{\mathbb{Q}}\left(H_{i}\left(I C_{\bullet}^{\bar{p}}(X ; \mathbb{Q})\right), H_{j}(C h(E \mathbb{Q}))\right) \\
& \cong \prod_{i-j=k} \operatorname{Hom}_{\mathbb{Q}}\left(I H_{i}^{\bar{p}}(X ; \mathbb{Q}), \pi_{j}(E \mathbb{Q})\right) \\
& \cong \prod_{i-j=k} \operatorname{Hom}_{\mathbb{Q}}\left(I H_{i}^{\bar{p}}(X ; \mathbb{Q}), \pi_{j}(E) \otimes \mathbb{Q}\right) .
\end{aligned}
$$

Remark 8.3. Alternatively, one may also obtain such universal coefficient isomorphisms as follows. Let $R$ be a commutative $S$-algebra and let $M, N$ be $R$-modules. Then there is a natural spectral sequence of differential $R_{*}$-modules

$$
E_{2}^{p, q}=\operatorname{Ext}_{R^{*}}^{p, q}\left(M^{*}, N^{*}\right) \Longrightarrow \operatorname{Ext}_{R}^{p+q}(M, N) \text {. }
$$

This sequence is of standard cohomological type, lies in the right half-plane and 
converges conditionally; cf. [EKMM97, Theorem IV.4.1]. Now substitute $R=H \mathbb{Q}$, $M=\Phi I C_{\bullet}^{\bar{p}}=\Phi I C_{\bullet}^{\bar{p}}(X ; \mathbb{Q}), N=E \mathbb{Q}$. Since then

$$
\begin{gathered}
R^{*}=\pi_{-*}(H \mathbb{Q})=\mathbb{Q}[0], \\
M^{*}=\pi_{-*}\left(\Phi I C_{\bullet}^{\bar{p}}\right) \cong H_{-*}\left(I C_{\bullet}^{\bar{p}}\right)=I H_{-*}^{\bar{p}}(X ; \mathbb{Q}),
\end{gathered}
$$

and

$$
N^{*}=\pi_{-*}(E \mathbb{Q}) \cong \pi_{-*}(E) \otimes \mathbb{Q},
$$

the $E_{2}$-term becomes

$$
E_{2}^{p, q}=\operatorname{Ext}_{\mathbb{Q}}^{p, q}\left(I H_{-*}^{\bar{p}}(X ; \mathbb{Q}), \pi_{-*}(E) \otimes \mathbb{Q}\right) .
$$

As $I H_{-*}^{\bar{p}}(X ; \mathbb{Q})$ is free over $\mathbb{Q}$, we have

$$
\operatorname{Ext}_{\mathbb{Q}}^{p, *}\left(I H_{-*}^{\bar{p}}(X ; \mathbb{Q}), \pi_{-*}(E) \otimes \mathbb{Q}\right)=0
$$

for $p>0$. Hence, the spectral sequence collapses to its edge-homomorphism. As for the target of the spectral sequence,

$$
\operatorname{Ext}_{R}^{p+q}(M, N)=N_{R}^{p+q}(M)=(E \mathbb{Q})_{H \mathbb{Q}}^{p+q}\left(\Phi I C_{\bullet}^{\bar{p}}\right)=I E_{\bar{p}}^{p+q}(X ; \mathbb{Q}) .
$$

\section{Künneth Spectral Sequence}

Theorem 9.1. Let $\bar{p}$ be a perversity such that

$$
\bar{p}(k)+\bar{p}(l) \leqslant \bar{p}(k+l) \leqslant \bar{p}(k)+\bar{p}(l)+2
$$

for all $k, l$, for instance, $\bar{p}=\bar{m}$. For any $S$-algebra $E$ and compact pseudomanifolds $X^{n}, Y^{m}$, there is a spectral sequence of the form

$$
E_{p, q}^{2}=\operatorname{Tor}_{p, q}^{E_{*} \otimes \mathbb{Q}}\left(I E_{*}^{\bar{p}}(X ; \mathbb{Q}), I E_{*}^{\bar{p}}(Y ; \mathbb{Q})\right) \Longrightarrow I E_{p+q}^{\bar{p}}(X \times Y ; \mathbb{Q}) .
$$

Proof. Let $U$ and $V$ denote the top strata of $X$ and $Y$, respectively. Let $\pi_{X}: X \times Y \rightarrow X, \pi_{U}: U \times V \rightarrow U, \pi_{Y}: X \times Y \rightarrow Y$ and $\pi_{V}: U \times V \rightarrow V$ denote the factor projections. By [CGJ92, Proposition 2], the multiplication morphism

$$
\pi_{U}^{*} \mathbb{Q}_{U}[n] \otimes \pi_{V}^{*} \mathbb{Q}_{V}[m] \longrightarrow \mathbb{Q}_{U \times V}[n+m]
$$

extends uniquely to an isomorphism

$$
\pi_{X}^{*} \mathbf{I} \mathbf{C}_{\bar{p}}^{\bullet}(X) \otimes \pi_{Y}^{*} \mathbf{I} \mathbf{C}_{\bar{p}}^{\bullet}(Y) \cong \mathbf{I} \mathbf{C}_{\bar{p}}^{\bullet}(X \times Y)
$$

in $\mathcal{D}^{b}(X \times Y)$ when $\bar{p}$ satisfies inequality (22). By [ $\left.\mathbf{B}^{+} \mathbf{8 4}, \mathrm{V} .10 .19\right]$, and using the compactness of $X$ and $Y$,

$$
R \Gamma\left(X \times Y ; \pi_{X}^{*} \mathbf{I} \mathbf{C}_{\bar{p}}^{\bullet}(X) \otimes \pi_{Y}^{*} \mathbf{I C}_{\bar{p}}^{\bullet}(Y)\right) \cong R \Gamma\left(X ; \mathbf{I C}_{\bar{p}}^{\bullet}(X)\right) \otimes R \Gamma\left(Y ; \mathbf{I C}_{\bar{p}}^{\bullet}(Y)\right) .
$$

Consequently, we obtain an Eilenberg-Zilber type isomorphism

$$
I C_{\bullet}^{\bar{p}}(X ; \mathbb{Q}) \otimes I C_{\bullet}^{\bar{p}}(Y ; \mathbb{Q}) \cong I C_{\bullet}^{\bar{p}}(X \times Y ; \mathbb{Q}) .
$$

Recall ([EKMM97]) that given any $S$-algebra $R$, right $R$-module $M$, and left $R$ module $N$, there is an Eilenberg-Moore type spectral sequence

$$
E_{p, q}^{2}=\operatorname{Tor}_{p, q}^{E_{*}(R)}\left(E_{*}(M), E_{*}(N)\right) \Longrightarrow E_{p+q}\left(M \wedge_{R} N\right) .
$$

Taking $R=H \mathbb{Q}, M=\Phi I C \bar{\bullet}(X ; \mathbb{Q}), N=\Phi I C \bar{\bullet}(Y ; \mathbb{Q})$, and using Corollary 2.4, we 
obtain a spectral sequence

$$
\operatorname{Tor}_{p, q}^{E_{*}(H \mathbb{Q})}\left(I E_{*}^{\bar{p}}(X ; \mathbb{Q}), I E_{*}^{\bar{p}}(Y ; \mathbb{Q})\right) \Longrightarrow E_{p+q}\left(\Phi I C_{\bullet}^{\bar{p}}(X ; \mathbb{Q}) \wedge_{H \mathbb{Q}} \Phi I C_{\bullet}^{\bar{p}}(Y ; \mathbb{Q})\right) .
$$

The target is

$$
\begin{aligned}
E_{p+q}\left(\Phi I C_{\bullet}^{\bar{p}}(X ; \mathbb{Q}) \wedge_{H \mathbb{Q}} \Phi I C_{\bullet}^{\bar{p}}(Y ; \mathbb{Q})\right) & \cong E_{p+q}\left(\Phi\left(I C_{\bullet}^{\bar{p}}(X ; \mathbb{Q}) \otimes_{\mathbb{Q}} I C_{\bullet}^{\bar{p}}(Y ; \mathbb{Q})\right)\right) \\
& \cong E_{p+q}\left(\Phi I C_{\bullet}^{\bar{p}}(X \times Y ; \mathbb{Q})\right) \\
& \cong I E_{p+q}^{\bar{p}}(X \times Y ; \mathbb{Q}),
\end{aligned}
$$

again using Corollary 2.4.

When the perversity $\bar{p}$ does not satisfy inequality (22), then the Eilenberg-Zilber isomorphism (23) fails in general. An example, due to [CGJ92], is given by $X=c T^{2}=Y$, the cone on the 2 -torus, and a perversity $\bar{p}$ with $\bar{p}(3)=0$ and $\bar{p}(6)=3$. The Betti numbers for $I H_{*}^{\bar{p}}\left(c T^{2} ; \mathbb{Q}\right)$ are $(1,2,0,0)$. Therefore, the Betti numbers for $I H_{*}^{\bar{p}}\left(c T^{2} ; \mathbb{Q}\right) \otimes I H_{*}^{\bar{p}}\left(c T^{2} ; \mathbb{Q}\right)$ are $(1,4,4,0,0,0,0)$. However, $I H_{*}^{\bar{p}}\left(c T^{2} \times c T^{2} ; \mathbb{Q}\right) \cong$ $I H_{*}^{\bar{p}}\left(c\left(T^{2} * T^{2}\right) ; \mathbb{Q}\right)$ has Betti numbers $(1,4,0,0,0,0,0)$.

\section{Local Intersection $\boldsymbol{E}$-Homology}

Let $X^{n}$ be a stratified pseudomanifold. Every point $x \in X$ has an open neighborhood $U$ of the form $U=\mathbb{R}^{n-k} \times c^{\circ} L^{k-1}$, called a distinguished neighborhood, where $L$ is the link of the path component of the pure stratum that contains $x$. The ordinary intersection homology of $U$ is well-known:

$$
I H_{r}^{\bar{p}}(U ; \mathbb{Q}) \cong \begin{cases}I H_{r-n+k-1}^{\bar{p}}(L ; \mathbb{Q}), & r \geqslant n-\bar{p}(k), \\ 0, & r<n-\bar{p}(k) .\end{cases}
$$

For any $S$-module $E$ and integer $a$, let $\tau_{\leqslant a} E$ denote the $(a+1)$-coconnective truncation of $E$, that is,

$$
\pi_{i}\left(\tau_{\leqslant a} E\right)= \begin{cases}\pi_{i}(E), & i \leqslant a, \\ 0, & i>a .\end{cases}
$$

With $E=H \mathbb{Z}$, the local result (24) may then be more succinctly formulated as

$$
I(H \mathbb{Z})_{r}^{\bar{p}}(U ; \mathbb{Q}) \cong I\left(\tau_{\leqslant r+\bar{p}(k)-n} H \mathbb{Z}\right)_{r-n+k-1}^{\bar{p}}(L ; \mathbb{Q}) .
$$

This formula generalizes to arbitrary coefficients $E$ :

Proposition 10.1. Let $X^{n}$ be a stratified pseudomanifold. Let $U=\mathbb{R}^{n-k} \times c^{\circ} L^{k-1}$ be an open distinguished neighborhood of a point in $X$, where $L$ is the link at that point. Then

$$
I E_{r}^{\bar{p}}(U ; \mathbb{Q}) \cong I\left(\tau_{\leqslant r+\bar{p}(k)-n} E\right)_{r-n+k-1}^{\bar{p}}(L ; \mathbb{Q})
$$

Proof. Let $U_{0}$ be the punctured neighborhood $U_{0}=\mathbb{R}^{n-k} \times\left(c^{\circ} L^{k-1}-\{c\}\right)$. Let $i$ : $U_{0} \hookrightarrow U$ be the open inclusion. Using a homeomorphism $U_{0} \cong \mathbb{R}^{n-k+1} \times L$, we have a projection $\pi: U_{0} \rightarrow L$ and the inclusion $j: L \hookrightarrow U_{0}$ at $0 \in \mathbb{R}^{n-k+1}$. Let $\mathbf{I}^{\bullet} \in \mathcal{D}^{b}\left(U_{0}\right)$ 
be an injective constructible sheaf complex. There is a natural morphism

$$
\rho: R \pi_{*} \mathbf{I}^{\bullet}=\pi_{*} \mathbf{I}^{\bullet} \longrightarrow j^{*} \mathbf{I}^{\bullet}
$$

that assigns to a section $s$ of $\pi_{*} \mathbf{I}^{\bullet}$ over an open subset $V \subset L$, which is by definition a section of $\mathbf{I}^{\bullet}$ over $\pi^{-1}(V) \cong \mathbb{R}^{n-k+1} \times V$, the section $\rho(s)$ obtained by restricting $s$ to $\{0\} \times V \subset \mathbb{R}^{n-k+1} \times V$. This morphism is a quasi-isomorphism; see, e.g., [Ban02, Chapter 5, Section 4.2]. Then the induced quasi-isomorphism

$$
R \Gamma(L ; \rho): R \Gamma\left(L ; \pi_{*} \mathbf{I}^{\bullet}\right) \longrightarrow R \Gamma\left(L ; j^{*} \mathbf{I}^{\bullet}\right)
$$

defines an isomorphism

$$
R \Gamma\left(U ; R i_{*} \mathbf{I}^{\bullet}\right) \cong R \Gamma\left(L ; j^{*} \mathbf{I}^{\bullet}\right)
$$

in $\mathcal{D}^{b}(\mathrm{pt})$ because

$$
R \Gamma\left(U ; R i_{*} \mathbf{I}^{\bullet}\right)=R \Gamma\left(U_{0} ; \mathbf{I}^{\bullet}\right)=R \Gamma\left(L ; \pi_{*} \mathbf{I}^{\bullet}\right),
$$

where we have used that $i_{*} \mathbf{I}^{\bullet}$ and $\pi_{*} \mathbf{I}^{\bullet}$ are both complexes of injective sheaves. If one specializes to $\mathbf{I}^{\bullet}$ an injective incarnation of $\mathbf{I C}_{\bar{p}}^{\bullet}\left(U_{0}\right)$, then this isomorphism induces an isomorphism

$$
I C_{\bullet}^{\bar{p}}(U ; \mathbb{Q})[n-k+1] \cong \tau_{\geqslant k-\bar{p}(k)-1} I C_{\bullet}^{\bar{p}}(L ; \mathbb{Q})
$$

in $\mathcal{D}(\mathbb{Q})$. On rational chain complexes, $-\otimes C h(E \mathbb{Q})$ is an exact functor and hence preserves quasi-isomorphisms. Consequently,

$$
I C_{\bullet}^{\bar{p}}(U ; \mathbb{Q})[n-k+1] \otimes C h(E \mathbb{Q}) \cong \tau_{\geqslant k-\bar{p}(k)-1} I C_{\bullet}^{\bar{p}}(L ; \mathbb{Q}) \otimes C h(E \mathbb{Q})
$$

in $\mathcal{D}(\mathbb{Q})$. Thus, with $a=r-n+k-1, \quad b=k-\bar{p}(k)-1$,

$$
\begin{aligned}
I E_{r}^{\bar{p}}(U ; \mathbb{Q}) & =\pi_{r}\left(\Phi I C_{\bullet}^{\bar{p}}(U ; \mathbb{Q}) \wedge_{H \mathbb{Q}} E \mathbb{Q}\right) \\
& =H_{r}\left(I C_{\bullet}^{\bar{p}}(U ; \mathbb{Q}) \otimes_{\mathbb{Q}} C h(E \mathbb{Q})\right) \\
& =H_{a+n-k+1}\left(I C_{\bullet}^{\bar{p}}(U ; \mathbb{Q}) \otimes_{\mathbb{Q}} C h(E \mathbb{Q})\right) \\
& =H_{a}\left(I C_{\bullet}^{\bar{p}}(U ; \mathbb{Q})[n-k+1] \otimes_{\mathbb{Q}} C h(E \mathbb{Q})\right) \\
& \cong H_{a}\left(\tau_{\geqslant b} I C_{\bullet}^{\bar{p}}(L ; \mathbb{Q}) \otimes C h(E \mathbb{Q})\right) \\
& \cong H_{a}\left(I C_{\bullet}^{\bar{p}}(L ; \mathbb{Q}) \otimes \tau_{\leqslant a-b} C h(E \mathbb{Q})\right) \\
& \cong \pi_{a}\left(\Phi I C_{\bullet}^{\bar{p}}(L ; \mathbb{Q}) \wedge_{H \mathbb{Q}} \tau_{\leqslant a-b} E \mathbb{Q}\right) \\
& =\pi_{r-n+k-1}\left(\Phi I C_{\bullet}^{\bar{p}}(L ; \mathbb{Q}) \wedge_{H \mathbb{Q}} \tau_{\leqslant r+\bar{p}(k)-n} E \mathbb{Q}\right) \\
& =I\left(\tau_{\leqslant r+\bar{p}(k)-n} E\right)_{r-n+k-1}^{\bar{p}}(L ; \mathbb{Q}) .
\end{aligned}
$$

\section{Self-Dual Theories on Non-Witt Spaces}

Let $X^{n}$ be a closed, $n$-dimensional, topological pseudomanifold. Clearly, the methods of this paper apply not only to the intersection chain sheaf $\mathbf{I C}_{\bar{p}}^{\bullet}(X) \in \mathcal{D}^{b}(X)$, but more generally to any differential complex $\mathbf{S}^{\bullet} \in \mathcal{D}^{b}(X)$ of sheaves of rational vector spaces on $X$. Indeed, $R f_{*} \mathbf{S}^{\bullet}$, where $R f_{*}: \mathcal{D}^{b}(X) \rightarrow \mathcal{D}^{b}(\mathrm{pt})$ is induced by $f: X \rightarrow$ pt, is a cochain complex and we set

$$
C^{\bullet}\left(X ; \mathbf{S}^{\bullet}\right)=R f_{*} \mathbf{S}^{\bullet} \text { and } C \bullet\left(X ; \mathbf{S}^{\bullet}\right)=C^{-\bullet}\left(X ; \mathbf{S}^{\bullet}\right) .
$$


Definition 11.1. Let $E$ be any $S$-module. The rational $E$-homology $E_{k}\left(X ; \mathbf{S}^{\bullet}\right)$ of $X$ with coefficients in a sheaf complex $\mathbf{S}^{\bullet}$ is defined to be

$$
E_{k}\left(X ; \mathbf{S}^{\bullet}\right)=(E \mathbb{Q})_{k}^{H \mathbb{Q}}\left(\Phi C \bullet\left(X ; \mathbf{S}^{\bullet}\right)\right) .
$$

The rational E-cohomology $E^{k}\left(X ; \mathbf{S}^{\bullet}\right)$ of $X$ with coefficients in a sheaf complex $\mathbf{S}^{\bullet}$ is defined to be

$$
E^{k}\left(X ; \mathbf{S}^{\bullet}\right)=(E \mathbb{Q})_{H \mathbb{Q}}^{k}\left(\Phi C \bullet\left(X ; \mathbf{S}^{\bullet}\right)\right) .
$$

Thus, $E_{k}\left(X ; \mathbf{I C}_{\bar{p}}^{\bullet}(X)\right)=I E_{k}^{\bar{p}}(X ; \mathbb{Q})$ and $E^{k}\left(X ; \mathbf{I C}_{\bar{p}}^{\bullet}(X)\right)=I E_{\bar{p}}^{k}(X ; \mathbb{Q})$.

Now assume that $X$ is oriented and $\mathbf{S}^{\bullet}$ is self-dual, that is, we are given an isomorphism $d: D_{X} \mathbf{S}^{\bullet}[n] \stackrel{\simeq}{\longrightarrow} \mathbf{S}^{\bullet}$ in $\mathcal{D}^{b}(X)$. Then $d$ will induce duality between the associated $E$-homology and $E$-cohomology groups:

Theorem 11.2. For a sheaf complex $\mathbf{S}^{\bullet} \in \mathcal{D}^{b}(X)$, a self-duality isomorphism $D_{X} \mathbf{S}^{\bullet}[n] \cong \mathbf{S}^{\bullet}$ induces a generalized Poincaré duality isomorphism

$$
E_{n-k}\left(X ; \mathbf{S}^{\bullet}\right) \cong E^{k}\left(X ; \mathbf{S}^{\bullet}\right)
$$

for any $S$-module $E$.

Proof. The proof of Theorem 5.3 carries over with $\mathbf{I C}_{\bar{p}}^{\bullet}(X)$ and $\mathbf{I C}_{\bar{q}}^{\bullet}(X)$ both replaced by $\mathbf{S}^{\bullet}$.

If $X$ has only strata of even codimension, then $\mathbf{I C}_{\bar{m}}^{\bullet}(X)$, the intersection chain sheaf with respect to the lower middle perversity $\bar{m}$, is self-dual, since $\mathbf{I C}_{\bar{m}}^{\bullet}(X)=\mathbf{I C}_{\bar{n}}^{\bullet}(X)$, the intersection chain sheaf with respect to the upper middle perversity $\bar{n}$. More generally, $\mathbf{I C}_{\bar{m}}^{\bullet}(X)$ is still self-dual on $X$ if $X$ is a Witt space. (For a Witt space, the middle-dimensional, lower middle perversity intersection homology of all links of odd-codimensional strata is required to vanish.) If $X$ is not a Witt space, then the canonical morphism $\mathbf{I} \mathbf{C}_{\bar{m}}^{\bullet}(X) \rightarrow \mathbf{I} \mathbf{C}_{\bar{n}}^{\bullet}(X)$ is not an isomorphism and $\mathbf{I C}_{\bar{m}}^{\bullet}(X)$ is not self-dual. We will briefly review some relevant results of [Ban02], where a theory of generalized Poincaré duality for non-Witt spaces is developed. Fix a stratification

$$
X=X_{n} \supset X_{n-2} \supset X_{n-3} \supset \cdots \supset X_{0} \supset X_{-1}=\varnothing
$$

such that $X_{j}$ is closed in $X$ and every non-empty $X_{j}-X_{j-1}$ is an open manifold of dimension $j$. Set $U_{k}=X-X_{n-k}$. As shown in [Ban02] and [Ban06], invariants of non-Witt spaces such as, e.g., $L$-classes, emerge from objects of a certain full subcategory $S D(X) \subset \mathcal{D}_{c}^{b}(X)$, where $\mathcal{D}_{c}^{b}(X)$ denotes the derived category of bounded, constructible differential complexes of sheaves of rational vector spaces. The objects of $S D(X)$ satisfy two properties: On the one hand, they are self-dual, on the other hand, they are as close to the middle perversity intersection chain sheaves as possible, that is, they interpolate between $\mathbf{I C}_{\bar{m}}^{\bullet}(X)$ and $\mathbf{I C}_{\bar{n}}^{\bullet}(X)$. The precise definition is as follows:

Definition 11.3. Let $S D(X)$ be the full subcategory of $\mathcal{D}_{c}^{b}(X)$ whose objects $\mathbf{S}^{\bullet}$ satisfy the following axioms:

(SD1) Top stratum normalization: $\left.\mathbf{S}^{\bullet}\right|_{U_{2}} \cong \mathbb{Q}_{U_{2}}[n]$.

(SD2) Lower bound: $\mathbf{H}^{i}\left(\mathbf{S}^{\bullet}\right)=0$, for $i<-n$. 
(SD3) Stalk condition for the upper middle perversity $\bar{n}$ : $\mathbf{H}^{i}\left(\left.\mathbf{S}^{\bullet}\right|_{U_{k+1}}\right)=0$, for $i>\bar{n}(k)-n, k \geqslant 2$.

(SD4) Self-Duality: $\mathbf{S}^{\bullet}$ has an associated isomorphism $d: \mathcal{D} \mathbf{S}^{\bullet}[n] \stackrel{\simeq}{\rightrightarrows} \mathbf{S}^{\bullet}$ such that $\mathcal{D} d[n]= \pm d$.

Here, $\mathbf{H}^{i}\left(\mathbf{S}^{\bullet}\right)$ denotes the cohomology sheaf of the complex $\mathbf{S}^{\bullet}$. Depending on $X$, the category $S D(X)$ may or may not be empty. The main structure theorem on $S D(X)$ is a description as a Postnikov system with fibers given by categories of Lagrangian structures along the strata of odd codimension:

Theorem 11.4. Let $n=\operatorname{dim} X$ be even. There is an equivalence of categories $S D(X) \simeq \operatorname{Lag}\left(U_{n}-U_{n-1}\right) \rtimes \operatorname{Lag}\left(U_{n-2}-U_{n-3}\right) \rtimes \cdots \rtimes \operatorname{Lag}\left(U_{4}-U_{3}\right) \rtimes \operatorname{Const}\left(U_{2}\right)$.

(Similarly for $n$ odd.)

In particular, there is a functor that assigns to every Lagrangian structure $\mathcal{L}$ a sheaf $\mathbf{I} \mathbf{C}_{\mathcal{L}}^{\bullet} \in S D(X)$ and every $\mathbf{S}^{\bullet} \in S D(X)$ is of this form.

Example 11.5. Let $X^{6}$ be the product of a circle with the (unreduced) suspension of complex projective space, $X^{6}=S^{1} \times \Sigma \mathbb{P}^{2}$. This space has a stratum of odd codimension 5 consisting of the disjoint union of two circles. The link of this stratum is $\mathbb{P}^{2}$ and there is no Lagrangian subspace in the middle cohomology $H^{2}\left(\mathbb{P}^{2}\right)$ (e.g., the signature $\left.\sigma\left(\mathbb{P}^{2}\right)=1 \neq 0\right)$. Then the structure theorem implies $S D\left(X^{6}\right)=\varnothing$, so there is no meaningful way to define intersection homology type invariants on $X^{6}$.

Let $X^{4}$ be the product of a circle with the suspension of a torus, $X^{4}=S^{1} \times \Sigma T^{2}$. The stratum of odd codimension 3 consists again of the disjoint union of two circles, but with link $T^{2}$. There are many Lagrangian subspaces $\mathcal{L}$ in the middle cohomology $H^{1}\left(T^{2}\right)$, and the structure theorem implies $S D\left(X^{4}\right) \neq \varnothing$. There is a self-dual sheaf $\mathbf{I C}_{\mathcal{L}}^{\bullet}$ on $X^{4}$ for every choice of $\mathcal{L}$.

Definition 11.6. Let $E$ be any $S$-module and $\mathcal{L}$ a Lagrangian structure along the strata of odd codimension of $X$. The rational $\mathcal{L}$-intersection $E$-homology $I E_{k}^{\mathcal{L}}(X ; \mathbb{Q})$ of $X$ is defined to be

$$
I E_{k}^{\mathcal{L}}(X ; \mathbb{Q})=E_{k}\left(X ; \mathbf{I C}_{\mathcal{L}}^{\bullet}\right)
$$

The rational $\mathcal{L}$-intersection $E$-cohomology $I E_{\mathcal{L}}^{k}(X ; \mathbb{Q})$ of $X$ is defined to be

$$
I E_{\mathcal{L}}^{k}(X ; \mathbb{Q})=E^{k}\left(X ; \mathbf{I C}_{\mathcal{L}}^{\bullet} .\right.
$$

Theorem 11.7. For every Lagrangian structure $\mathcal{L}$, there exists a factorization

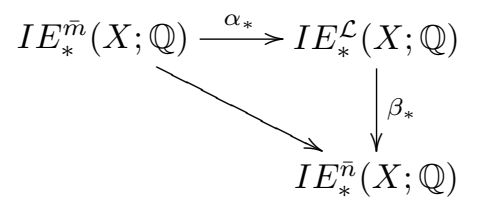


of the canonical morphism such that the duality square

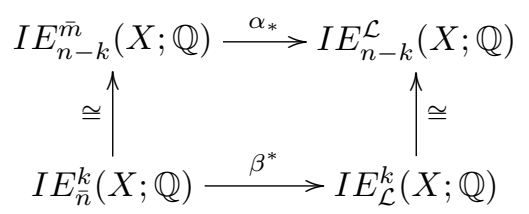

commutes.

Proof. Given any $\mathbf{I C}_{\mathcal{L}}^{\bullet} \in S D(X)$, there exists a factorization

$$
\mathbf{I C}_{\bar{m}}^{\bullet}(X) \stackrel{\alpha}{\longrightarrow} \mathbf{I C}_{\mathcal{L}}^{\bullet} \stackrel{\beta}{\longrightarrow} \mathbf{I C}_{\bar{n}}^{\bullet}(X)
$$

of the canonical morphism $\mathbf{I C}_{\bar{m}}^{\bullet}(X) \rightarrow \mathbf{I} \mathbf{C}_{\bar{n}}^{\bullet}(X)$ such that

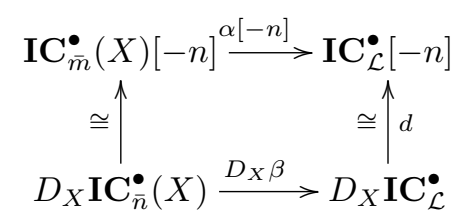

(where $d$ is given by (SD4)) commutes, by [Ban02, Theorem 2.2]. The proof will now consist of applying the techniques and identities of the proof of Theorem 5.3 to this square. Thus, applying $R f_{*}$, for $f: X \rightarrow$ pt, to the square, passing from cochain to chain complexes, and tensoring with $C h(E \mathbb{Q})$, the diagram becomes

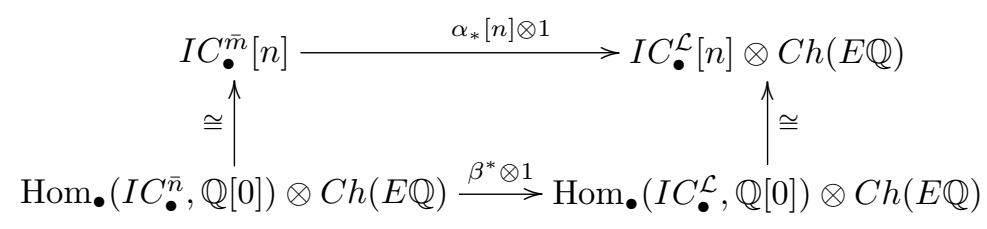

in $\mathcal{D}(\mathbb{Q})$. Note that on rational chain complexes, $-\otimes C h(E \mathbb{Q})$ is an exact functor and hence preserves quasi-isomorphisms. Next, apply homology $H_{-k}(-)$ :

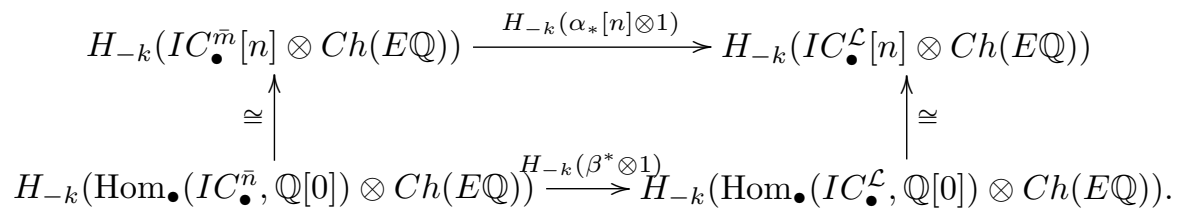

Let us briefly write $C_{\bullet}^{*}=\operatorname{Hom}_{\bullet}\left(C_{\bullet}, \mathbb{Q}[0]\right)$ for a chain complex $C_{\bullet}$, and $E_{\bullet}=C h(E \mathbb{Q})$. The proof of Theorem 5.3 gives isomorphisms

$$
\begin{aligned}
& H_{-k}\left(I C_{\bullet}^{\bar{m}}[n] \otimes E_{\bullet}\right) \stackrel{\cong}{\longrightarrow} I E_{n-k}^{\bar{m}}(X ; \mathbb{Q}), \quad H_{-k}\left(I C_{\bullet}^{\mathcal{L}}[n] \otimes E_{\bullet}\right) \stackrel{\cong}{\longrightarrow} I E_{n-k}^{\mathcal{L}}(X ; \mathbb{Q}), \\
& H_{-k}\left(I C_{\bullet}^{\bar{n} *} \otimes E_{\bullet}\right) \stackrel{\cong}{\longrightarrow} I E_{\bar{n}}^{k}(X ; \mathbb{Q}), \quad H_{-k}\left(I C_{\bullet}^{\mathcal{L} *} \otimes E_{\bullet}\right) \stackrel{\cong}{\longrightarrow} I E_{\mathcal{L}}^{k}(X ; \mathbb{Q}) .
\end{aligned}
$$

Using these, we define maps

$$
I E_{n-k}^{\bar{m}}(X ; \mathbb{Q}) \stackrel{\alpha_{*}}{\longrightarrow} I E_{n-k}^{\mathcal{L}}(X ; \mathbb{Q}), \quad I E_{\bar{n}}^{k}(X ; \mathbb{Q}) \stackrel{\beta^{*}}{\longrightarrow} I E_{\mathcal{L}}^{k}(X ; \mathbb{Q}),
$$


by

$$
\begin{gathered}
I E_{n-k}^{\bar{m}}(X ; \mathbb{Q}) \cong H_{-k}\left(I C_{\bullet}^{\bar{m}}[n] \otimes E_{\bullet}\right) \longrightarrow H_{-k}\left(I C_{\bullet}^{\mathcal{L}}[n] \otimes E_{\bullet}\right) \cong I E_{n-k}^{\mathcal{L}}(X ; \mathbb{Q}), \\
I E_{\bar{n}}^{k}(X ; \mathbb{Q}) \cong H_{-k}\left(I C_{\bullet}^{\bar{n} *} \otimes E_{\bullet}\right) \longrightarrow H_{-k}\left(I C_{\bullet}^{\mathcal{L} *} \otimes E_{\bullet}\right) \cong I E_{\mathcal{L}}^{k}(X ; \mathbb{Q}),
\end{gathered}
$$

respectively, and the duality isomorphisms

$$
I E_{\bar{n}}^{k}(X ; \mathbb{Q}) \stackrel{\cong}{\longrightarrow} I E_{n-k}^{\bar{m}}(X ; \mathbb{Q}), \quad I E_{\mathcal{L}}^{k}(X ; \mathbb{Q}) \stackrel{\cong}{\longrightarrow} I E_{n-k}^{\mathcal{L}}(X ; \mathbb{Q})
$$

are given by the compositions

$$
\begin{aligned}
& I E_{\bar{n}}^{k}(X ; \mathbb{Q}) \cong H_{-k}\left(I C_{\bullet}^{\bar{n} *} \otimes E_{\bullet}\right) \cong H_{-k}\left(I C_{\bullet}^{\bar{\bullet}}[n] \otimes E_{\bullet}\right) \cong I E_{n-k}^{\bar{m}}(X ; \mathbb{Q}), \\
& I E_{\mathcal{L}}^{k}(X ; \mathbb{Q}) \cong H_{-k}\left(I C_{\bullet}^{\mathcal{L} *} \otimes E_{\bullet}\right) \cong H_{-k}\left(I C_{\bullet}^{\mathcal{L}}[n] \otimes E_{\bullet}\right) \cong I E_{n-k}^{\mathcal{L}}(X ; \mathbb{Q}),
\end{aligned}
$$

respectively. We obtain a cube

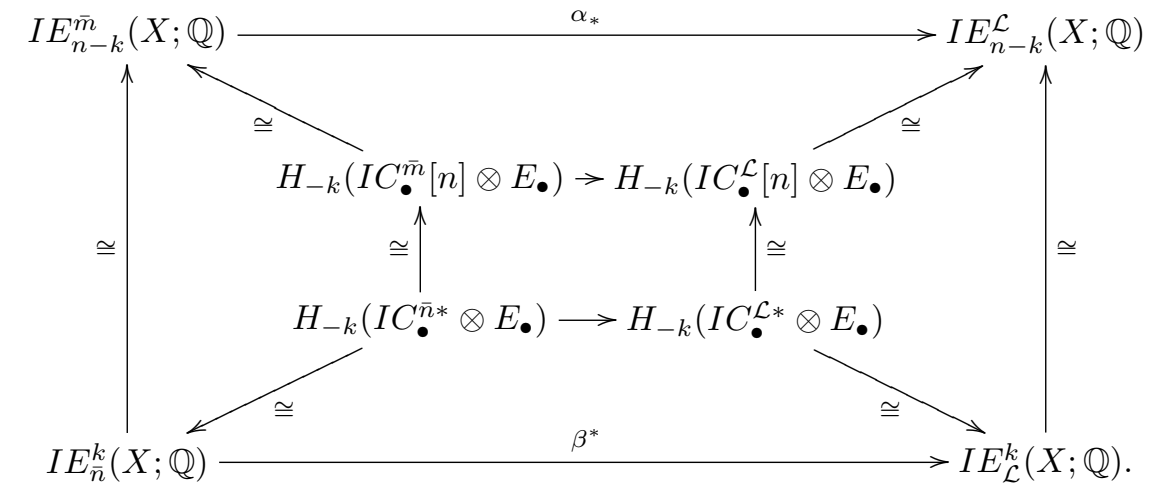

The five interior faces of the cube commute by definition. Thus, the sixth, outer face commutes as well.

\section{An Example}

Let $\left[x_{0}: \cdots: x_{5}\right]$ denote homogeneous coordinates for complex projective space $\mathbb{P}^{5}$. With

$$
Q(x)=x_{0}^{4}+\cdots+x_{5}^{4}
$$

we define the quartic 4 -fold

$$
V=\{Q(x)=0\} \subset \mathbb{P}^{5} .
$$

For $C(x)=x_{0} x_{3}-x_{1} x_{2}$, set

$$
X=\{C(x)=0\} \subset V .
$$

This quadric hypersurface in $V$ is a Calabi-Yau 3-fold. It is singular at $x_{0}=x_{1}=x_{2}=$ $x_{3}=0$, as there $d C(x)$ vanishes. Thus, $X$ has precisely the 4 singularities $[0: 0: 0: 0:$ $\omega: 1], \omega^{4}=-1$, all of which are nodes. Let $U \cong \mathbb{C}^{4}$ be a small open neighborhood in $V$ of such a node. Then the neighborhood $U \cap X$ of the node in $X$ is a complex cone over $\mathbb{P}^{1} \times \mathbb{P}^{1}$, i.e., $\{u v-w t=0\} \subset \mathbb{C}^{4}$. The real picture is that of a real cone over $S^{2} \times S^{3}$. (Physicists call $X$ a "conifold" because it has only isolated conical 
singularities.) Let $k u$ be the connective, complex $K$-theory spectrum ( $S$-algebra). We wish to calculate the intersection $k u$-homology Poincaré series

$$
I k u P_{t}(X)=\sum_{i} t^{i} \operatorname{dim} I k u_{i}^{\bar{m}}(X ; \mathbb{Q})
$$

as well as the intersection $k u$-cohomology Poincaré series

$$
I k u P^{t}(X)=\sum_{i} t^{i} \operatorname{dim} I k u_{\bar{m}}^{i}(X ; \mathbb{Q})
$$

of $X$. Throughout, we shall rely on data from [Hüb92]. Let $\left[y_{0}: y_{1}\right]$ be homogeneous coordinates for $\mathbb{P}^{1}$. Define three hypersurfaces in $\mathbb{P}^{5} \times \mathbb{P}^{1}$ by

$$
\begin{aligned}
& V_{1}=\{Q(x)=0\}, \\
& V_{2}=\left\{x_{0} y_{0}+x_{1} y_{1}=0\right\}, \\
& V_{3}=\left\{x_{2} y_{0}+x_{3} y_{1}=0\right\} .
\end{aligned}
$$

Let $Y$ be the complete intersection

$$
\begin{aligned}
Y & =V_{1} \cap V_{2} \cap V_{3} & & \text { in } \mathbb{P}^{5} \times \mathbb{P}^{1} \\
& =V_{2} \cap V_{3} & & \text { in } V \times \mathbb{P}^{1} .
\end{aligned}
$$

Then $Y$ is a real 6 -dimensional Calabi-Yau manifold. Let $d_{11}=4, d_{21}=0$ be the degrees of $Q$ in $x$ and $y$, respectively. Let $d_{12}=1, d_{22}=1$ be the degrees of the defining equation of $V_{2}$ in $x$ and $y$ respectively, and let $d_{13}=1, d_{23}=1$ be the degrees of the defining equation of $V_{3}$ in $x$ and $y$ respectively. Let

$$
J_{1}=c_{1}\left(\mathcal{O}_{\mathbb{P}^{5}}(1)\right), \quad J_{2}=c_{1}\left(\mathcal{O}_{\mathbb{P}^{1}}(1)\right)
$$

be positive $(1,1)$-forms on $\mathbb{P}^{5}$ and $\mathbb{P}^{1}$ respectively, representing the first Chern classes of the hyperplane bundles so that the total Chern classes are given by

$$
c\left(\mathbb{P}^{5}\right)=\left(1+J_{1}\right)^{6}, \quad c\left(\mathbb{P}^{1}\right)=\left(1+J_{2}\right)^{2} .
$$

Define

$$
A=\frac{1}{3} \sum_{r, s, t=1}^{2}\left(\delta^{r s t}\left(n_{r}+1\right)-\sum_{j=1}^{3} d_{r j} d_{s j} d_{t j}\right) J_{r} J_{s} J_{t}
$$

where $n_{1}=5, n_{2}=1$ are the dimensions of the two projective spaces, and

$$
B=\bigwedge_{j=1}^{3}\left(d_{1 j} J_{1}+d_{2 j} J_{2}\right)
$$

Then the Euler characteristic $\chi(Y)$ can be computed by integrating the third Chern class of $Y$ :

$$
\chi(Y)=\int_{Y} c_{3}(Y)=[A \cdot B]_{\mathrm{top}}
$$

where $[-]_{\text {top }}$ means the coefficient of $J_{1}^{5} J_{2}$. Working out $A$ and $B$ yields

$$
A=-20 J_{1}^{3}-2 J_{1}^{2} J_{2}, \quad B=4\left(J_{1}^{3}+2 J_{1}^{2} J_{2}\right)
$$


so the coefficient of $J_{1}^{5} J_{2}$ in $A \cdot B$, and hence $\chi(Y)$, is -168 . The general form of the Hodge diamond of a Calabi-Yau manifold of dimension 3 is

$\begin{array}{cccccccc} & & & 1 & & & \\ & & 0 & & & 0 & & \\ & & b_{2,1} & b_{1,1} & & 0 & \\ & 0 & & b_{1,1} & & 0 & \\ & & 0 & & 0 & & \\ & & & 1 & & & \end{array}$

Since

$$
\chi(Y)=\sum_{r=0}^{6}(-1)^{r} b_{r}, \quad b_{r}=\sum_{p=0}^{r} b_{p, r-p},
$$

we have the relation

$$
\chi(Y)=2\left(b_{1,1}-b_{2,1}\right) .
$$

Iterative application of the Lefschetz-Bott hyperplane theorem [Bot59] gives $b_{1,1}=2$. Thus,

$$
b_{2,1}=b_{1,1}-\frac{1}{2} \chi=86
$$

and $b_{3}=2+2 b_{2,1}=174$. Thus, the Hodge diamond of $Y$ is

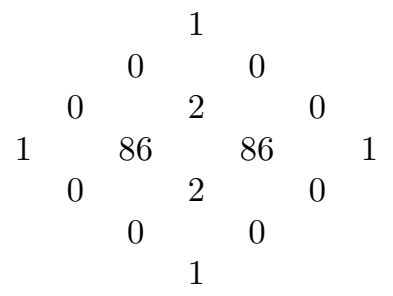

and

$$
H P_{t}(Y)=1+2 t^{2}+174 t^{3}+2 t^{4}+t^{6} .
$$

The projection $V \times \mathbb{P}^{1} \longrightarrow V$ along $\mathbb{P}^{1}$ induces a map $f: Y \rightarrow X$. This map is a Kähler small resolution. For a space $Z$, let $E P_{t}(Z)$ denote the rational $E$-homology Poincaré series of $Z$. By Theorem 6.2,

$$
\operatorname{IkuP}_{t}(X)=k u P_{t}(Y) .
$$

By the Atiyah-Hirzebruch spectral sequence,

$$
k u P_{t}(Y)=H P_{t}(Y) \cdot k u P_{t}(\mathrm{pt})
$$

The homotopy groups of the connective complex $K$-theory spectrum are

$$
\pi_{i}(k u)= \begin{cases}\mathbb{Z}, & n \geqslant 0 \text { and even } \\ 0, & n \text { otherwise }\end{cases}
$$


SO

$$
k u P_{t}(\mathrm{pt})=\sum_{i=0}^{\infty} t^{2 i}
$$

Thus,

$$
k u P_{t}(Y)=\frac{1+2 t^{2}+174 t^{3}+2 t^{4}+t^{6}}{1-t^{2}}
$$

and for the intersection $k u$-homology,

$$
I k u P_{t}(X)=1+3 t^{2}+174 t^{3}+5 t^{4}+174 t^{5}+6 \sum_{i=3}^{\infty} t^{2 i}+174 \sum_{i=3}^{\infty} t^{2 i+1} .
$$

The corresponding cohomological series can now be computed by the Poincaré duality Theorem 5.3:

$$
I k u P^{t}(X)=\sum_{i} t^{i} \operatorname{dim} I k u_{6-i}^{\bar{m}}(X ; \mathbb{Q})=\sum_{j} t^{6-j} \operatorname{dim} I k u_{j}^{\bar{m}}(X ; \mathbb{Q})=t^{6} \cdot I k u P_{1 / t}(X) .
$$

Hence,

$$
I k u P^{t}(X)=t^{6}+3 t^{4}+174 t^{3}+5 t^{2}+174 t+6 \sum_{i=0}^{\infty} t^{-2 i}+174 \sum_{i=0}^{\infty} t^{-2 i-1} .
$$

Note that by [GM83],

$$
I H P_{t}(X)=H P_{t}(Y)=1+2 t^{2}+174 t^{3}+2 t^{4}+t^{6} .
$$

This gives another way to compute the cohomological intersection $k u$-theory series, using the Atiyah-Hirzebruch formula, Proposition 8.2: If

$$
\operatorname{IkuP} P^{t}(X)=\sum_{i} c_{i} t^{i}
$$

then

$$
I k u_{\bar{m}}^{i}(X ; \mathbb{Q}) \cong \prod_{p-q=i} \operatorname{Hom}_{\mathbb{Q}}\left(I H_{p}^{\bar{m}}(X ; \mathbb{Q}), \pi_{q}(k u) \otimes_{\mathbb{Z}} \mathbb{Q}\right)
$$

implies

$$
c_{i}=\sum_{p-q=i} \operatorname{dim} I H_{p}^{\bar{m}}(X ; \mathbb{Q}) \cdot \operatorname{dim}\left(\pi_{q}(k u) \otimes_{\mathbb{Z}} \mathbb{Q}\right)=\sum_{q=0}^{\infty} \operatorname{dim} I H_{2 q+i}^{\bar{m}}(X ; \mathbb{Q}) .
$$

Using (26), we obtain

$c_{6}=1, c_{5}=0, c_{4}=3, c_{3}=174, c_{2}=5, c_{1}=174, c_{0}=6, c_{-1}=174, c_{-2}=6, \ldots$ in accordance with (25).

\section{References}

$\left[\mathbf{B}^{+} \mathbf{8 4}\right] \quad$ A. Borel et al., Intersection cohomology, Progr. Math. 50, Birkhäuser Verlag, Boston, 1984. 
[Ban02] M. Banagl, Extending intersection homology type invariants to nonWitt spaces, Mem. Amer. Math. Soc. 160 (2002), no. 760, 1-83.

[Ban06] _ The $L$-class of non-Witt spaces, Ann. of Math. (2) 163 (2006), no. $3,743-766$.

[Ban07] _ Topological invariants of stratified spaces, Springer Monographs in Mathematics, Springer-Verlag, Berlin Heidelberg, 2007.

[Bot59] R. Bott, On a theorem of Lefschetz, Michigan Math. J. 6 (1959), 211216.

[CGJ92] D. C. Cohen, M. Goresky, and L. Ji, On the Künneth formula for intersection cohomology, Trans. Amer. Math. Soc. 333 (1992), no. 1, 63-69.

[CSW91] S. E. Cappell, J. L. Shaneson, and S. Weinberger, Classes topologiques caractéristiques pour les actions de groupes sur les espaces singuliers, C. R. Acad. Sci. Paris Sér. I Math. 313 (1991), 293-295.

[EKMM97] A. D. Elmendorf, I. Kriz, M. A. Mandell, and J. P. May, Rings, modules, and algebras in stable homotopy theory, Mathematical Surveys and Monographs, vol. 47, Amer. Math. Soc., 1997.

[GM80] M. Goresky and R. D. MacPherson, Intersection homology theory, Topology 19 (1980), 135-162.

[GM83] _ Intersection homology II, Invent. Math. 71 (1983), 77-129.

[Hüb92] T. Hübsch, Calabi-Yau Manifolds: A Bestiary for Physicists, World Scientific, Singapore, 1992.

[LJMS86] L. G. Lewis Jr., J. P. May, and M. Steinberger, Equivariant stable homotopy theory, Lecture Notes in Math., no. 1213, Springer Verlag, 1986.

[Rob83] A. Robinson, Derived tensor products in stable homotopy theory, Topology 22 (1983), no. 1, 1-18.

[Rob87] _ The extraordinary derived category, Math. Z. 196 (1987), 231-238.

[Wal65] C. T. C. Wall, Finiteness conditions for CW complexes II, Proc. $R$. Soc. Lond. A 295 (1965), 129-139.

Markus Banagl banagl@mathi.uni-heidelberg.de

Mathematisches Institut, Universität Heidelberg, Im Neuenheimer Feld 288, 69120 Heidelberg, Germany 